\title{
Natural transformations of tensor algebras and representations of combinatorial groups
}

\author{
JELENA GRBIĆ
}

JIE WU

\begin{abstract}
Natural linear and coalgebra transformations of tensor algebras are studied. The representations of certain combinatorial groups are given. These representations are connected to natural transformations of tensor algebras and to the groups of the homotopy classes of maps from the James construction to loop spaces. Applications to homotopy theory appear in a sequel [4].
\end{abstract}

16W30; 20F38, 55P35

\section{Introduction}

This paper has as its motivation a problem coming from classical homotopy theory, namely, the study of the natural maps from loop suspensions to loop spaces. The method we propose for analyzing properties of certain unstable maps departs from classical unstable homotopy theoretical constructions. We apply the homology functor to natural maps from loop suspensions, and obtain certain functorial coalgebra transformations This approach justifies the primarily goal of the paper, which is the study of the algebra of natural linear transformations of tensor algebra and related groups of natural coalgebra transformations. These algebras and groups are studied by means of combinatorial group theory. We start with an analogue of a non-commutative exterior algebra defined by Cohen [1] and define several new combinatorial algebras as its generalizations. These algebras are then identified with the corresponding algebras of natural linear transformations of tensor algebras. On the other hand, looking at certain subgroups of the group of units of these combinatorial algebras, we recover the Cohen group $K_{n}$ and obtain its generalizations (see Section 2 for the definitions). We proceed by establishing group isomorphisms between these combinatorial groups and the corresponding groups of natural coalgebra transformations.

Combinatorial groups, on their own, are closely related to the motivating problem, that is, to the group of natural maps from loop suspensions to loop spaces. This connection is explained in more detail in the sequel to this paper [4]. There we present 
solutions to some problems in classical homotopy theory concerning natural maps from loop suspensions to loop spaces obtained by applying the algebraic machinery developed in this paper. Related calculations are done by exploiting the rich structure in combinatorial groups.

Before outlining the main results achieved in the paper, let us explaining the geometrical motivation behind doing the algebra by quoting the main results of the successive paper.

Recall that for any pointed space $X$, the James-Hopf map $H_{k}: J(X) \longrightarrow J\left(X^{(k)}\right)$ is combinatorially defined by

$$
H_{k}\left(x_{1} x_{2} \ldots x_{n}\right)=\prod_{1 \leq i_{1}<i_{2}<\cdots<i_{k} \leq n}\left(x_{i_{1}} x_{i_{2}} \ldots x_{i_{k}}\right)
$$

with right lexicographical order in the product. The $n$-th fold Samelson product $\widetilde{W}_{n}$ on $X$ is given by the composite

$$
\widetilde{W}_{n}: X \wedge \ldots \wedge X \stackrel{E \wedge \ldots \wedge E}{\longrightarrow} \Omega \Sigma X \wedge \ldots \wedge \Omega \Sigma X \stackrel{[[,], \ldots,]}{\longrightarrow} \Omega \Sigma X
$$

where $E: X \longrightarrow \Omega \Sigma X$ is the canonical inclusion and the second map $[[],, \ldots$,$] is$ the $n$-fold commutator. The $n$-fold Whitehead product $W_{n}$ on $X$ is defined as the adjoint of the $n$-fold Samelson product $\widetilde{W}_{n}$.

Theorem A Let $X$ be a pointed space with the null homotopic reduced diagonal $\bar{\Delta}: X \longrightarrow X \wedge X$. Then for $n>k$,

$$
J\left(X^{(n)}\right) \stackrel{\Omega W_{n}}{\longrightarrow} J(X) \stackrel{H_{k}}{\longrightarrow} J\left(X^{(k)}\right)
$$

is null homotopic if $k$ does not divide $n$.

The result concerning the Barratt Conjecture can be formulated as follows.

Theorem B Let $X=\Sigma X^{\prime}$ be a suspension and let $f: X \longrightarrow \Omega Y$ be a map such that $p^{r}[f]=0$ in the group $[X, \Omega Y]$. Let $J(f): J(X) \longrightarrow \Omega Y$ be the canonical multiplicative extension of $f$. Then the following hold.

(1) The map $\left.\quad J(f)\right|_{J_{n}(X)}: J_{n}(X) \longrightarrow \Omega Y$

has order $p^{r+t}$ in $\left[J_{n}(X), \Omega Y\right]$ if $n<p^{t+1}$.

(2) The composite

$$
J_{p^{t+1}}(X) \stackrel{\left.J(f)\right|_{J_{p^{t+1}}(X)} ^{\longrightarrow}}{\longrightarrow} \Omega Y \stackrel{p^{r+t}}{\longrightarrow} \Omega Y
$$

is homotopic to the composite 
$J_{p^{t+1}}(X) \stackrel{\text { pinch }}{\longrightarrow} X^{\left(p^{t+1}\right)} \stackrel{p^{r-1}\left(\sum_{\tau \in \Sigma_{p} t+1} 1 \wedge \tau\right)}{\longrightarrow} X^{\left(p^{t+1}\right)} \stackrel{\widetilde{W}_{p^{t+1}}}{\longrightarrow} J(X) \stackrel{J(f)}{\longrightarrow} \Omega Y$,

where $p^{r+t}: \Omega Y \longrightarrow \Omega Y$ is the $p^{r+t}$-th power map, $\widetilde{W}_{n}$ is the $n$-fold Samelson product and

$1 \wedge \tau\left(x_{1} \wedge \cdots \wedge x_{p^{t+1}}\right)=x_{1} \wedge x_{\tau(2)} \wedge \cdots \wedge x_{\tau\left(p^{t+1}\right)}: X^{\left(p^{t+1}\right)} \longrightarrow X^{\left(p^{t+1}\right)}$

is the map which permutes positions.

(3) Let $g=J(f) \circ \widetilde{W}_{p^{t+1}} \circ\left(\sum_{\tau \in \Sigma_{p^{t+1}-1}} 1 \wedge \tau\right) \circ p^{r-1}: X^{\left(p^{t+1}\right)} \longrightarrow \Omega Y$. Then $g$ is an equivariant map with respect to the symmetric group action, that is,

$$
g \circ \sigma \simeq g \text { for any } \sigma \in \Sigma_{p^{t+1}} .
$$

Now we are ready to outline the main results in this paper.

Through the course of the paper $R$ will be a commutative ring with identity unless specified differently. By $\operatorname{Hom}_{R}(C, A)$ we denote the algebra of natural linear transformations from a coalgebra $C$ to an algebra $A$, with the multiplication given by the convolution product. The convolution product $f * g$ of $f, g: C \longrightarrow A$ is defined by

$$
C \stackrel{\psi}{\longrightarrow} C \otimes C \stackrel{f \otimes g}{\longrightarrow} A \otimes A \stackrel{\mu}{\longrightarrow} A
$$

where $\psi: C \longrightarrow C \otimes C$ is the comultiplication and $\mu: A \otimes A \longrightarrow A$ is the multiplication.

Let $V$ be a free $R$-module. The James (coalgebra) filtration $\left\{J_{n}(V)\right\}_{n \geq 0}$ of the tensor algebra $T(V)$ is defined by

$$
J_{n}(V)=\bigoplus_{j \leq n} T_{j}(V)
$$

for $n \geq 0$, where $T_{j}(V)=V^{\otimes j}$, the $j^{t h}$ stage of the tensor length filtration for $T(V)$. Let $C(V)=J_{1}(V)$.

A non-commutative analogue of an exterior algebra is given by $A^{R}\left(y_{1}, y_{2}, \cdots, y_{n}\right)$ the quotient algebra of the tensor algebra $T\left(y_{1}, \cdots, y_{n}\right)$ over $R$ modulo the two sided ideal generated by the monomials $y_{i_{1}} \cdots y_{i_{t}}$ with $i_{p}=i_{q}$ for some $1 \leq p<q \leq t$.

Proposition 1.1 There is an isomorphism of algebras

$$
\theta_{n}: A^{R}\left(y_{1}, \cdots, y_{n}\right) \longrightarrow \operatorname{Hom}_{R}\left(C(-)^{\otimes n}, T(-)\right) .
$$


Furthermore, the James filtration $\left\{J_{n}(V)\right\}_{n \geq 0}$ induces a cofiltration of algebras

$$
\operatorname{Hom}_{R}(T(-), T(-)) \rightarrow \cdots \rightarrow \operatorname{Hom}_{R}\left(J_{n}(-), T(-)\right) \rightarrow \cdots \rightarrow \operatorname{Hom}_{R}\left(J_{0}(-), T(-)\right)
$$

where the algebra $\operatorname{Hom}_{R}(T(-), T(-))$ is given by the inverse limit

$$
\operatorname{Hom}_{R}(T(-), T(-)) \cong \lim _{n} \operatorname{Hom}_{R}\left(J_{n}(-), T(-)\right) .
$$

Let $L_{n}^{R}$ be the equalizer of the projection maps

$$
\pi_{j}: A^{R}\left(y_{1}, \cdots, y_{n}\right) \longrightarrow A^{R}\left(y_{1}, \cdots, y_{n-1}\right)
$$

for $1 \leq j \leq n$. Then we have the following result.

Proposition 1.2 The map

$$
\theta_{n}=\left.\theta_{n}\right|_{L_{n}^{R}}: L_{n}^{R} \longrightarrow \operatorname{Hom}_{R}\left(J_{n}(-), T(-)\right)
$$

is an algebra isomorphism for $n \geq 0$.

For two $R$-modules $C$ and $D$, define their smash product $C \wedge D$ to be the quotient module

$$
C \wedge D=(C \otimes D) /\left(C \otimes_{R} R \oplus R \otimes_{R} D\right) .
$$

Proposition 1.3 There are combinatorial algebras $A_{n}^{R}[k],{ }^{R} L_{n}^{(l)}$ and ${ }^{R} L_{n}^{(l),(k)}$ (for their definitions see Section 3) such that there are algebra isomorphisms

(1) $\left.\operatorname{Hom}_{R}\left(C(-)^{\otimes n}\right), T\left(-^{\wedge k}\right)\right) \cong A_{n}^{R}[k]$

(2) $\operatorname{Hom}_{R}\left(J_{n}\left(-{ }^{\otimes l}\right), T(-)\right) \cong{ }^{R} L_{n}^{(l)}$

(3) $\operatorname{Hom}_{R}\left(J_{n}(-\otimes l), T\left(-{ }^{\otimes k}\right)\right) \cong{ }^{R} L_{n}^{(l),(k)}$

for $1 \leq n \leq \infty$.

Let $\operatorname{Coalg}(C, D)$ denote the group of natural coalgebra transformations from a coalgebra $C$ to a Hopf algebra $D$ with the multiplication given by the convolution product. The James filtration $\left\{J_{n}(-)\right\}_{n \geq 0}$ induces a cofiltration of the progroup Coalg $(T(-), T(-))$. Recall that $C(-)=J_{1}(-)$.

In [6], Selick and Wu described some properties of the groups Coalg $(T(-), T(-))$ and Coalg $\left(C(-)^{\otimes n}, T(-)\right)$. By the following theorems, we extend their results identifying the groups of natural coalgebra transformations of the James filtration $\left\{J_{n}(-)\right\}_{n \geq 0}$ and their new generalizations with combinatorial groups introduced in Section 1. 
Theorem 1.4 There is an isomorphism of groups

$$
e: K_{n}^{R} \longrightarrow \operatorname{Coalg}\left(C(-)^{\otimes n}, T(-)\right) \quad \text { for } n \geq 0 .
$$

Define $\mathcal{H}_{n}^{R}$ to be the equalizer of the projection homomorphisms

$$
p_{j}: K_{n}^{R} \longrightarrow K_{n-1}^{R} \quad \text { for } 1 \leq j \leq n .
$$

Theorem 1.5 There is an isomorphism of groups

$$
e: \mathcal{H}_{n}^{R} \longrightarrow \operatorname{Coalg}\left(J_{n}(-), T(-)\right) \text { for } 1 \leq n \leq \infty .
$$

Having in mind the problem solved in Theorem A, we define a generalisation $K_{n}^{R}(k)$ of $K_{n}$. We set an algebraic notation which is motivated by geometry. Let $\left\{x_{i_{1}}\left|x_{i_{2}}\right| \cdots \mid x_{i_{k}}\right\}$ be a notation for a word of length $\mathrm{k}$, in letters $x_{i_{1}}, x_{i_{2}}, \ldots, x_{i_{k}}$. In a successive paper these words will be related to the composite

$$
X^{n} \stackrel{p_{i_{1} \cdots i_{k}}}{\longrightarrow} X^{(k)} \stackrel{E}{\longrightarrow} J\left(X^{(k)}\right) .
$$

Let $G$ be a set consisting of all the words $\left\{x_{i_{1}}\left|x_{i_{2}}\right| \cdots \mid x_{i_{k}}\right\}$ with $1 \leq i_{j} \leq n$ for $1 \leq j \leq k$ such that $i_{s} \neq i_{t}$ for all $1 \leq s<t \leq k$.

The group $K_{n}^{R}(k)$ is defined combinatorially for any commutative ring $R$ so that the generators are elements of $G$ and a certain set of relations, which will be discussed in detail later on in the paper. For $k=1$, we denote $K_{n}^{R}(1)$ by $K_{n}^{R}$. We will call $K_{n}^{R}(k)$ a Cohen group as it is a generalization of the combinatorial group $K_{n}=K_{n}^{\mathbb{Z}}(1)$ defined by Cohen [1].

Theorem 1.6 There are combinatorial groups $K_{n}^{R}(k),{ }^{R} \mathcal{H}_{n}^{(l)}$ and ${ }^{R} \mathcal{H}_{n}^{(l),(k)}$ (see Section 1 for their definitions) such that there are group isomorphisms

(1) $\left.\operatorname{Coalg}\left(C(-)^{\otimes n}\right), T\left(-^{\wedge k}\right)\right) \cong K_{n}^{R}(k)$

(2) Coalg $\left(J_{n}\left(-{ }^{\otimes l}\right), T(-)\right) \cong R_{\mathcal{H}_{n}^{(l)}}$

(3) $\operatorname{Coalg}\left(J_{n}\left(-{ }^{\otimes l}\right), T\left(-{ }^{\otimes k}\right)\right) \cong{ }^{R} \mathcal{H}_{n}^{(l),(k)}$

for $1 \leq n \leq \infty$.

The compositions of the group isomorphisms in Theorems 1.4, 1.5 and 1.6, with the canonical inclusion of the natural coalgebra transformations $\operatorname{Coalg}(C, D)$ of tensor algebras into the natural linear transformations $\operatorname{Hom}_{R}(C, D)$ of tensor algebras give rise to faithful representations of the introduced combinatorial groups to the corresponding algebras of natural linear transformations of tensor algebras. 
In the sequel to this paper [4] we establish a connection between the combinatorial groups $\mathcal{H}_{n}^{R},{ }^{R} \mathcal{H}_{n}^{(l)}$ and ${ }^{R} \mathcal{H}_{n}^{(l)(k)}$ and the groups of the homotopy classes of maps from the topological James construction $J(X)$ of spaces $X$ with the null homotopic reduced diagonal. We do that by first restricting the $\operatorname{ring} R$ to $\mathbb{Z}$ or $\mathbb{Z} / p^{r}$ and then constructing injective maps:

$$
\begin{gathered}
e_{X}: \mathcal{H}_{n}^{R} \longrightarrow\left[J_{n}(X), J(X)\right] \\
e_{X}:{ }^{R} \mathcal{H}_{n}^{(l)} \longrightarrow\left[J_{n}\left(X^{(l)}\right), J(X)\right] \\
e_{X}:{ }^{R} \mathcal{H}_{n}^{(l)(k)} \longrightarrow\left[J_{n}\left(X^{(l)}\right), J\left(X^{(k)}\right)\right]
\end{gathered}
$$

The disposition of the paper is as follows. Section 2 catalogues all the combinatorial groups of our study and states various properties they satisfy. Section 3 relates combinatorial algebras to natural linear transformations of tensor algebras. Section 4 builds up to and deals with the primary focus of the paper, that is, establishing group isomorphisms between the combinatorial groups defined in Section 2 and the corresponding groups of functorial coalgebra transformations of tensor algebras. Section 5 gives a representation of the combinatorial group $K_{n}^{R}(k)$ and relates that group to the group of certain functorial coalgebra transformations.

Acknowledgements The authors would like to thank Professors Fred Cohen, John Berrick and Paul Selick for their helpful suggestions and kind encouragement. The first author would also like to thank Professor John Berrick and the second author for making it possible for her to visit the National University of Singapore for a term and providing her with such a friendly working atmosphere.

\section{Cohen groups and their generalizations}

In $[2 ; 1]$, Cohen defined the combinatorial group $K_{n}^{R}\left(x_{1}, x_{2}, \ldots, x_{n}\right)$ for $R=\mathbb{Z}$ or $\mathbb{Z} / p^{r}$. Following his approach we define the Cohen group $K_{n}^{R}\left(x_{1}, x_{2}, \ldots, x_{n}\right)$ for any commutative ring $R$ with identity. Let $R_{x}$ denote a copy of $R$ labeled by $x$. Write $x^{r}$ for the element $r \in R_{x}=R$, and just $x$ for $x^{1}$.

Definition 2.1 The Cohen group $K_{n}^{R}\left(x_{1}, x_{2}, \ldots, x_{n}\right)$ is the quotient group of the free product $F_{n}^{R}=\bigsqcup_{i=1}^{n} R_{x_{i}}$ modulo the relations

(1) $\left.\left[\left[x_{i_{1}}^{r_{1}}, x_{i_{2}}^{r_{2}}\right], x_{i_{3}}^{r_{3}}\right], \cdots, x_{i_{l}}^{r_{l}}\right]=1$ if $i_{s}=i_{t}$ for some $1 \leq s, t \leq l$;

(2) $\left.\left.\left[\left[x_{i_{1}}^{r_{1}}, x_{i_{2}}^{r_{2}}\right], x_{i_{3}}^{r_{3}}\right], \cdots, x_{i_{l}}^{r_{l}}\right]=\left[\left[x_{i_{1}}^{r_{1}^{\prime}}, x_{i_{2}}^{r_{2}^{\prime}}\right], x_{i_{3}}^{r_{3}^{\prime}}\right], \cdots, x_{i_{l}}^{r_{l}^{\prime}}\right]$ if there is an equality $r_{1} \cdot r_{2} \cdot \ldots \cdot r_{l}=r_{1}^{\prime} \cdot r_{2}^{\prime} \cdot \ldots \cdot r_{l}^{\prime}$. 
where $[x, y]=x^{-1} y^{-1} x y$ and $\left.\left[\left[y_{1}, y_{2}\right], y_{3}\right], \cdots, y_{l}\right]$ is an iterated commutator.

For brevity of notation, we write $K_{n}^{R}$ for the Cohen group $K_{n}^{R}\left(x_{1}, x_{2}, \ldots, x_{n}\right)$ when the generators are assumed, and for $K_{n}^{\mathbb{Z}}$ we use Cohen's notation $K_{n}$. As we will show in the sequel, the Cohen group $K_{n}$ is closely related to the group $\left[X^{n}, J(X)\right]$ for any space $X$ such that its reduced diagonal $\bar{\Delta}: X \longrightarrow X \wedge X$ is null homotopic.

Define group homomorphisms $p_{j}: F_{n}^{R} \longrightarrow F_{n-1}^{R}$ and $s_{j}: F_{n-1}^{R} \longrightarrow F_{n}^{R}$ by

$$
p_{j}\left(x_{i}^{r}\right)=\left\{\begin{array}{ll}
x_{i}^{r} & \text { for } i<j \\
1 & \text { for } i=j \\
x_{i-1}^{r} & \text { for } i>j
\end{array} \quad s_{j}\left(x_{i}^{r}\right)= \begin{cases}x_{i}^{r} & \text { for } i<j \\
x_{i+1}^{r} & \text { for } i \geq j\end{cases}\right.
$$

for $1 \leq j \leq n$. It follows that the composites $F_{n}^{R} \stackrel{p_{j}}{\longrightarrow} F_{n-1}^{R} \longrightarrow K_{n-1}^{R}$ and $F_{n-1}^{R} \stackrel{s_{j}}{\longrightarrow} F_{n}^{R} \longrightarrow K_{n}^{R}$ factor through $K_{n}^{R}$ and $K_{n-1}^{R}$, respectively, inducing the homomorphisms of the Cohen groups $p_{j}: K_{n}^{R} \longrightarrow K_{n-1}^{R}$ and $s_{j}: K_{n-1}^{R} \longrightarrow K_{n}^{R}$.

The next objective is to generalise the Cohen groups $K_{n}$, by defining new combinatorial groups related to the group $\left[X^{n}, J\left(X^{(k)}\right)\right]$.

Definition 2.2 The group $K_{n}^{R}(k)$ is defined combinatorially as the quotient group of the free product

$$
\coprod_{\substack{1 \leq i_{j} \leq n \\ 1 \leq j \leq k}} R_{\left\{x_{i_{1}}\left|x_{i_{2}}\right| \cdots \mid x_{i_{k}}\right\}}
$$

modulo the relations given by the following identities:

(1) $\left\{x_{i_{1}}\left|x_{i_{2}}\right| \cdots \mid x_{i_{k}}\right\}^{r}=1 \quad$ if $i_{s}=i_{t}$ for some $1 \leq s<t \leq k$;

(2) $\left[\left[\left\{x_{i_{1}}\left|x_{i_{2}}\right| \cdots \mid x_{i_{k}}\right\}^{r_{1}},\left\{x_{i_{k+1}}\left|x_{i_{k+2}}\right| \cdots \mid x_{i_{2 k}}\right\}^{r_{2}}, \cdots\right.\right.$

$$
\left.\cdots,\left\{x_{i_{(l-1) k+1}}\left|x_{i_{(l-1) k+2}}\right| \cdots \mid x_{i_{l k}}\right\}^{r_{l}}\right]=1
$$

if $i_{s}=i_{t}$ for some $1 \leq s<t \leq k l$, where $\left[\left[a_{1}, a_{2}, \cdots, a_{l}\right]=\left[\left[a_{1}, a_{2}\right], a_{3}\right], \cdots, a_{l}\right]$ with $[x, y]=x^{-1} y^{-1} x y$;

(3) $\left[\left[\left\{x_{i_{1}}\left|x_{i_{2}}\right| \cdots \mid x_{i_{k}}\right\}^{r_{1}},\left\{x_{i_{k+1}}\left|x_{i_{k+2}}\right| \cdots \mid x_{i_{2 k}}\right\}^{r_{2}}, \cdots\right.\right.$,

$$
\begin{aligned}
& \left.\cdots,\left\{x_{i_{(l-1) k+1}}\left|x_{i_{(l-1) k+2}}\right| \cdots \mid x_{i_{l k}}\right\}^{r_{l}}\right]= \\
& {\left[\left[\left\{x_{i_{1}}\left|x_{i_{2}}\right| \cdots \mid x_{i_{k}}\right\}^{r_{1}^{\prime}},\left\{x_{i_{k+1}}\left|x_{i_{k+2}}\right| \cdots \mid x_{i_{2 k}}\right\}^{r_{2}^{\prime}, \cdots,}\right.\right.} \\
& \left.\cdots,\left\{x_{i_{(l-1) k+1}}\left|x_{i_{(l-1) k+2}}\right| \cdots \mid x_{i_{l k}}\right\}_{l}^{r_{l}^{\prime}}\right]
\end{aligned}
$$


Remark In a similar way as for the Cohen group $K_{n}^{R}$, whenever $R=\mathbb{Z}$ we will denote $K_{n}^{R}(k)$ by $K_{n}(k)$. It is obvious that for $k=1, K_{n}(1)$ is the Cohen group $K_{n}$.

Let $q$ be an integer. Notice that the group $K_{n}^{\mathbb{Z} / q}(k)$ is the quotient group of $K_{n}(k)$ modulo the following additional relations:

$$
\left\{x_{i_{1}}\left|x_{i_{2}}\right| \cdots \mid x_{i_{k}}\right\}^{q}=1
$$

for each generator $\left\{x_{i_{1}}\left|x_{i_{2}}\right| \cdots \mid x_{i_{k}}\right\}$.

Selick and $\mathrm{Wu}$ [6] defined combinatorial groups $\mathcal{H}_{n}^{R}$ in order to study the group $\left[J_{n}(X), J(X)\right]$ with the main goal of obtaining information on the group $[J(X), J(X)]$. It should be remarked that the concept (and notation) of $\mathcal{H}_{n}^{R}$ was invented by Cohen. Here we recall their definition.

Definition 2.3 The group $\mathcal{H}_{n}^{R}$ is defined to be the equalizer of the projections $p_{j}: K_{n}^{R}\left(x_{1}, x_{2}, \ldots, x_{n}\right) \longrightarrow K_{n-1}^{R}\left(x_{1}, x_{2}, \ldots, x_{n-1}\right)$ for $1 \leq j \leq n$.

By definition, as $\left.p_{i}\right|_{\mathcal{H}_{n}^{R}}=\left.p_{j}\right|_{\mathcal{H}_{n}^{R}}$ for $1 \leq i, j \leq n$, there is a homomorphism $d_{n}: \mathcal{H}_{n}^{R} \longrightarrow \mathcal{H}_{n-1}^{R}$ such that the diagram

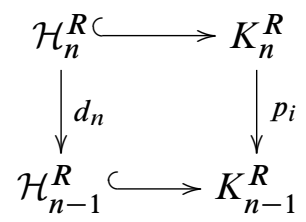

commutes for each $1 \leq i \leq n$.

The following lemma was proved by Selick and $\mathrm{Wu}[6]$. As the proof is illustrative and will be used later on in the paper, we include it here.

Lemma 2.4 The homomorphism $d_{n}: \mathcal{H}_{n}^{R} \longrightarrow \mathcal{H}_{n-1}^{R}$ is an epimorphism for each $n$.

Proof By induction on $k$, we show that $d_{k, n}=d_{k+1} \circ \ldots \circ d_{n}: \mathcal{H}_{n}^{R} \longrightarrow \mathcal{H}_{k}^{R}$ is an epimorphism for $k \leq n$. Clearly, $d_{1, n}$ is an epimorphism. Suppose that $d_{k-1, n}$ is an epimorphism with $k>1$ and let $\alpha \in \mathcal{H}_{k}^{R}$. Since $d_{k-1, n}: \mathcal{H}_{n}^{R} \longrightarrow \mathcal{H}_{k-1}^{R}$ is onto, we assume that $\alpha$ lies in the kernel of $d_{k}: \mathcal{H}_{k}^{R} \longrightarrow \mathcal{H}_{k-1}^{R}$. Let

$$
\alpha_{k, n}=\prod_{1 \leq i_{1}<i_{2}<\ldots<i_{n-k} \leq n} s_{i_{n-k}} s_{i_{n-k-1}} \ldots s_{i_{1}} \alpha \in K_{n}^{R}
$$

with lexicographic order from the right. Then it is routine to check that $\alpha_{k, n} \in \mathcal{H}_{n}^{R}$ with $d_{k, n}\left(\alpha_{k, n}\right)=\alpha$ and hence the result. 
Lemma 2.4 results in a progroup (a tower of group epimorphisms)

$$
\mathcal{H}^{R} \longrightarrow \cdots \longrightarrow \mathcal{H}_{n}^{R} \stackrel{d_{n}}{\longrightarrow} \mathcal{H}_{n-1}^{R} \longrightarrow \cdots \longrightarrow \mathcal{H}_{1}^{R}
$$

where $\mathcal{H}^{R}$ is the group defined by the inverse limit

$$
\mathcal{H}^{R}=\lim _{d_{n}} \mathcal{H}_{n}^{R}
$$

We recall an important description of the kernel of $d_{n}: \mathcal{H}_{n}^{R} \longrightarrow \mathcal{H}_{n-1}^{R}$ that will be used later on in the paper.

Let $R$ be a commutative ring and $\bar{V}$ the free $R$-module with basis $\left\{x_{1}, \ldots, x_{n}\right\}$. Then Lie $^{R}(n)$ denotes the $R$-submodule of $\bar{V} \otimes n$ generated by the $n$-fold commutators $\left[\left[x_{\sigma(1)}, x_{\sigma(2)}\right], \ldots, x_{\sigma(n)}\right]$ for $\sigma$ a permutation in the symmetric group $\Sigma_{n}$ on $n$ letters.

Theorem 2.5 (Cohen [1]) Let $\Lambda(n)$ be the kernel of the group homomorphism $d_{n}: \mathcal{H}_{n}^{R} \longrightarrow \mathcal{H}_{n-1}^{R}$. Then $\Lambda(n)$ is isomorphic to $\operatorname{Lie}^{R}(n)$ for $R=\mathbb{Z}$ or $\mathbb{Z} / p^{r}$.

In this paper we go a step further by defining new combinatorial groups and algebras in order to study natural transformations of tensor algebras. In a sequel we translate this algebraic setting into geometry which aims at solutions of certain problems of classical homotopy theory. With this in mind we first define two families of groups $R_{\mathcal{H}}^{(l)}$ and ${ }^{R} \mathcal{H}_{n}^{(l),(k)}$ that can be seen as generalizations of $\mathcal{H}_{n}^{R}$ and which will shed some light on the study of the groups $\left[J_{n}\left(X^{(l)}\right), J(X)\right]$ and $\left[J_{n}\left(X^{(l)}\right), J\left(X^{(k)}\right)\right]$.

The projection $p_{j}: K_{n}^{R}\left(x_{1}, x_{2}, \ldots, x_{n}\right) \longrightarrow K_{n-1}^{R}\left(x_{1}, x_{2}, \ldots, x_{n-1}\right)$ is given by

$$
p_{j}\left(x_{i}^{r}\right)= \begin{cases}x_{i}^{r} & \text { for } i<j \\ 1 & \text { for } i=j \\ x_{i-1}^{r} & \text { for } i>j\end{cases}
$$

for $1 \leq j \leq l$.

Define the projective homomorphism

$$
p_{j+\{1, \ldots, l\}}: K_{l n}^{R}\left(x_{1}, x_{2}, \ldots, x_{l n}\right) \longrightarrow K_{l(n-1)}^{R}\left(x_{1}, x_{2}, \ldots, x_{l(n-1)}\right)
$$

by

$$
p_{j+\{1, \ldots, l\}}\left(x_{i}^{r}\right)= \begin{cases}x_{i}^{r} & \text { for } i<j+1 \\ 1 & \text { for } j+1 \leq i \leq j+l \\ x_{i-1}^{r} & \text { for } i>j+l\end{cases}
$$

for $0 \leq j \leq n-1$. 
Definition 2.6 Define ${ }^{R} \mathcal{H}_{n}^{(l)}$ to be the equalizer of the projections

$$
\begin{gathered}
K_{l n}^{R}\left(x_{1}, x_{2}, \ldots, x_{l n}\right) \cap\left(\bigcap_{j=0}^{n-1}\left(\bigcap_{i=1}^{l} \operatorname{Ker} p_{j l+i}\right)\right) \\
p_{j+\{1, \ldots, l\}} \downarrow \cdot \downarrow \\
K_{l(n-1)}^{R}\left(x_{1}, x_{2}, \ldots, x_{l(n-1)}\right) \bigcap\left(\bigcap_{j=0}^{n-2}\left(\bigcap_{i=1}^{l} \operatorname{Ker} p_{j l+i}\right)\right)
\end{gathered}
$$

for $0 \leq j \leq n-1$,

As the group ${ }^{R} \mathcal{H}_{n}^{(l)}$ is given by the equalizer of the projections $p_{j+\{1, \ldots, l\}}$ for $0 \leq$ $j \leq n-1$, that is, $\left.p_{i+\{1, \ldots, l\}}\right|_{R \mathcal{H}_{n}^{(l)}}=\left.p_{j+\{1, \ldots, l\}}\right|_{R \mathcal{H}_{n}^{(l)}}$ for $0 \leq i, j \leq n-1$, there is a homomorphism $d_{n}:{ }^{R} \mathcal{H}_{n}^{(l)} \longrightarrow{ }^{R} \mathcal{H}_{n-1}^{(l)}$ such that the diagram

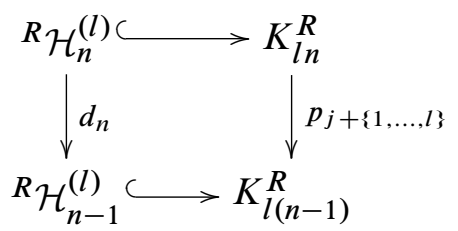

commutes for each $1 \leq j \leq n-1$.

Lemma 2.7 The homomorphism $d_{n}:{ }^{R} \mathcal{H}_{n}^{(l)} \longrightarrow{ }^{R} \mathcal{H}_{n-1}^{(l)}$ is an epimorphism for each $n$.

Proof Noticing that the homomorphism

$$
K_{l n}^{R} \bigcap\left(\bigcap_{j=0}^{n-1}\left(\bigcap_{i=1}^{l} \operatorname{Ker} p_{j l+i}\right)\right) \stackrel{p_{j}+\{1, \ldots, l\}}{\longrightarrow} K_{l(n-1)}^{R} \bigcap\left(\bigcap_{j=0}^{n-2}\left(\bigcap_{i=1}^{l} \operatorname{Ker} p_{j l+i}\right)\right)
$$

is an epimorphism for each $0 \leq j \leq n-1$, the proof follows along the lines of the proof of Lemma 2.4.

Lemma 2.7 results in a progroup

$$
R \mathcal{H}^{(l)} \longrightarrow \cdots \longrightarrow R \mathcal{H}_{n}^{(l)} \stackrel{d_{n}}{\longrightarrow} R \mathcal{H}_{n-1}^{(l)} \longrightarrow \cdots \longrightarrow R_{\mathcal{H}_{1}^{(l)}}
$$

where ${ }^{R} \mathcal{H}^{(l)}$ is the group defined by the inverse limit

$$
R \mathcal{H}^{(l)}=\lim _{d_{n}} R \mathcal{H}_{n}^{(l)} .
$$


Definition 2.8 The group ${ }^{R} \mathcal{H}_{n}^{(l),(k)}$ is the subgroup of $K_{l n}^{R}(k)$ given as the equalizer of the projections

$$
\begin{gathered}
K_{l n}^{R}(k) \cap\left(\bigcap_{j=0}^{n-1}\left(\bigcap_{i=1}^{l} \operatorname{Ker} p_{j l+i}\right)\right) \\
p_{j+\{1, \ldots, l\}} \mid \cdot \cdot \downarrow \\
K_{l(n-1)}^{R}(k) \cap\left(\bigcap_{j=0}^{n-2}\left(\bigcap_{i=1}^{l} \operatorname{Ker} p_{j l+i}\right)\right)
\end{gathered}
$$

for $0 \leq j \leq n-1$, where the homomorphisms $p_{j}$ and $p_{j+\{1, \ldots, l\}}$ are induced on the generalized Cohen group $K_{l n}^{R}(k)$ by the projections $p_{j+\{1, \ldots, l\}}$ on $K_{n l}^{R}$ defined by $(2-1)$.

In an analogous fashion as for $\mathcal{H}^{R}$ and ${ }^{R} \mathcal{H}^{(l)}$, using the fact that ${ }^{R} \mathcal{H}_{n}^{(l),(k)}$ is given as the equalizer of the projections $p_{j+\{1, \ldots, l\}}$, there are group epimorphisms

$$
d_{n}:{ }^{R} \mathcal{H}_{n}^{(l),(k)} \longrightarrow R^{R} \mathcal{H}_{n-1}^{(l),(k)}
$$

such that the diagram

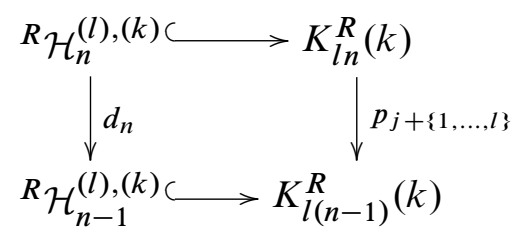

commutes for each $1 \leq j \leq n-1$. Thus there is a progroup

$$
R \mathcal{H}^{(l),(k) \longrightarrow \cdots \longrightarrow R} \mathcal{H}_{n}^{(l),(k)} \stackrel{d_{n}}{\longrightarrow} R \mathcal{H}_{n-1}^{(l),(k)} \longrightarrow \cdots \longrightarrow R_{\mathcal{H}_{1}^{(l),(k)}}^{\longrightarrow}
$$

where ${ }^{R} \mathcal{H}^{(l),(k)}$ is the group defined by the inverse limit

$$
R_{\mathcal{H}^{(l),(k)}}=\lim _{d_{n}} R_{\mathcal{H}_{n}^{(l),(k)}} .
$$

This introduces all the combinatorial groups we will consider in the paper.

\section{Combinatorial algebras and natural linear transformations of tensor algebras}

In this section the ground ring is assumed to be a commutative ring $R$ with identity. For a Hopf algebra $H$ over $R$, denote the comultiplication by $\psi: H \longrightarrow H \otimes H$; 
the multiplication by $\mu: H \otimes H \longrightarrow H$; the augmentation by $\epsilon: H \longrightarrow R$ and the coaugmentation by $\eta: R \longrightarrow H$.

Let $V$ be a free $R$-module. The James (coalgebra) filtration $\left\{J_{n}(V)\right\}_{n \geq 0}$ of the tensor algebra $T(V)$ is defined as an $R$-module by

$$
J_{n}(V)=\bigoplus_{j \leq n} T_{j}(V)
$$

for $n \geq 0$, where $T_{j}(V)$ is the $j^{t h}$ stage of the tensor word length filtration of $T(V)$. An coalgebra structure on the filtration is given by requiring that the elements of $V$ are primitive and then multiplicatively extend to all of $J_{n}(V)$. With this coalgebra structure $J_{n}(V)$ is a subcoalgebra of the primitively generated Hopf algebra $T(V)$.

Let $C$ be a (graded) coalgebra and let $A$ be a (graded) algebra. The convolution product $f * g$ of $f, g: C \longrightarrow A$ is defined by

$$
C \stackrel{\psi}{\longrightarrow} C \otimes C \stackrel{f \otimes g}{\longrightarrow} A \otimes A \stackrel{\mu}{\longrightarrow} A
$$

where $\psi: C \longrightarrow C \otimes C$ is the comultiplication and $\mu: A \otimes A \longrightarrow A$ is the multiplication.

Let $\operatorname{Hom}_{R}(T(-), T(-))$ denote the set of all functorial $R$-linear maps from $T(V)$ to itself. The convolution product induces a multiplication in $\operatorname{Hom}_{R}(T(-), T(-))$ under which $\operatorname{Hom}_{R}(T(-), T(-))$ becomes an algebra over $R$. Furthermore, the James filtration

$$
J_{0}(V) \subseteq J_{1}(V) \subseteq \cdots \subseteq J_{n}(V) \subseteq \cdots \subseteq T(V)
$$

induces a cofiltration of algebras

$$
\operatorname{Hom}_{R}(T(-), T(-)) \rightarrow \cdots \rightarrow \operatorname{Hom}_{R}\left(J_{n}(-), T(-)\right) \rightarrow \cdots \rightarrow \operatorname{Hom}_{R}\left(J_{0}(-), T(-)\right) .
$$

The purpose of this section is on the one hand to describe connections between combinatorial algebras and natural linear transformations of tensor algebras, and on the other hand to establish the context in which an already developed machinery can be utilized to study connections between the combinatorial groups $K_{n}^{R}, \mathcal{H}_{n}^{R},{ }^{R} \mathcal{H}_{n}^{(l)}$ and ${ }^{R} \mathcal{H}_{n}^{(l)(k)}$ with certain groups of functorial coalgebra transformations of $T(-)$ and $J_{n}(-)$. We start by recalling Cohen's definition [1] of a non-commutative analogue of the exterior algebra on $\left\{y_{1}, y_{2}, \cdots, y_{n}\right\}$.

Definition 3.1 The Cohen algebra $A^{R}\left(y_{1}, y_{2}, \cdots, y_{n}\right)$ is defined as the quotient algebra of the tensor algebra $T\left(y_{1}, \cdots, y_{n}\right)$ over $R$ modulo the two sided ideal generated by the monomials $y_{i_{1}} \cdots y_{i_{t}}$ with $i_{p}=i_{q}$ for some $1 \leq p<q \leq t$. 
Remark As in the case of the Cohen group $K_{n}^{R}$, Cohen [1] gave the definition of $A^{R}\left(y_{1}, \cdots, y_{n}\right)$ for $R=\mathbb{Z}$ or $\mathbb{Z} / p^{r}$, while in our case $R$ can be any commutative ring with identity.

The following result, which Cohen stated for $R=\mathbb{Z}$ or $\mathbb{Z} / p^{r}$ in [1, Theorem 1.3.2], also holds for an arbitrary commutative ring $R$.

Proposition 3.2 The algebra $A^{R}\left(y_{1}, \cdots, y_{n}\right)$ is a graded algebra over $R$ and in degree $t, A\left(y_{1}, \cdots, y_{n}\right)_{t}$ is a free $R$-module with basis $y_{i_{\sigma(1)}} \cdots y_{i_{\sigma(t)}}$ where $\sigma \in \Sigma_{t}$ acts on $\{1,2, \cdots, t\}$ and $1 \leq i_{1}<i_{2}<\cdots<i_{t} \leq n$.

Proof The proof follows directly from the definition of $A^{R}\left(y_{1}, \cdots, y_{n}\right)$.

Recall that $C(V)$ is defined as $J_{1}(V)$.

Definition 3.3 Let $V$ be a free $R$-module. The algebra $A_{n}(V)$ is defined to be the subalgebra (under convolution) of $\operatorname{Hom}_{R}\left(C(V)^{\otimes n}, T(V)\right)$ generated by the elements $y_{j}$ given by the composites

$$
y_{j}: C(V)^{\otimes n} \stackrel{p_{j}}{\longrightarrow} C(V) \stackrel{q}{\longrightarrow} V \stackrel{E}{\longrightarrow} T(V),
$$

for $1 \leq j \leq n$, where $p_{j}: C(V)^{\otimes n} \longrightarrow C(V)$ is given by

$$
p_{j}\left(x_{1} \otimes x_{2} \otimes \cdots \otimes x_{n}\right)=\epsilon\left(x_{1}\right) \cdots \epsilon\left(x_{j-1}\right) x_{j} \epsilon\left(x_{j+1}\right) \cdots \epsilon\left(x_{n}\right),
$$

$q: C(V) \longrightarrow V$ is the projection and $E: V \longrightarrow T(V)$ is the inclusion.

Lemma 3.4 Let $V$ be a connected graded free $R$-module. Then, in the algebra $A_{n}(V)$, the following equation holds for the generators $y_{j}$ :

$$
y_{i_{1}} y_{i_{2}} \cdots y_{i_{t}}=0
$$

if $i_{j}=i_{k}$ for some $j \neq k$.

Proof The lemma can be easily proved by looking at its geometrical realisation. There exists a space $X=\vee S^{n_{\alpha}}$ with $n_{\alpha} \geq 1$ such that $\widetilde{H}_{*}(X ; R)=V$. Thus $H_{*}(X ; R) \cong C(V)$ as coalgebras and $p_{j}: C(V)^{\otimes n} \longrightarrow C(V)$ is given by

$$
\left(p_{j}\right)_{*}: H_{*}\left(X^{n} ; R\right) \longrightarrow H_{*}(X ; R),
$$

where $p_{j}: X^{n} \longrightarrow X$ is the $j$-th projection. Thus $y_{i_{1}} \cdots y_{i_{t}}$ is represented by the composite

$$
H_{*}\left(X^{n} ; R\right) \stackrel{\left(p_{i_{1} \cdots i_{t}}\right)_{*}}{\longrightarrow} H_{*}\left(X^{t} ; R\right) \stackrel{\gamma}{\longrightarrow} \widetilde{H}_{*}\left(X^{(t)} ; R\right)=V^{\otimes t C} \longrightarrow T(V) .
$$


where $p_{i_{1} \cdots i_{t}}\left(x_{1}, \cdots, x_{n}\right)=\left(x_{i_{1}}, \cdots, x_{i_{t}}\right)$ and $\gamma: X^{t} \longrightarrow X^{(t)}$ is the quotient map. Notice that if $i_{j}=i_{k}$ for some $j \neq k$, then there exists a map $f: X^{n} \longrightarrow X^{(t-1)}$ such that

$$
\bar{\Delta} \circ f=\gamma \circ p_{i_{1} \cdots i_{t}}: X^{n} \longrightarrow X^{(t)}
$$

where $\bar{\Delta}: X^{(t-1)} \longrightarrow X^{(t)}$ is some reduced diagonal map. Notice that

$$
(\bar{\Delta})_{*}: \widetilde{H}_{*}\left(X^{(t-1)} ; R\right) \longrightarrow \widetilde{H}_{*}\left(X^{(t)} ; R\right)
$$

is zero since $X$ is a suspension. The assertion of the lemma follows.

Corollary 3.5 Let $V$ be a connected graded free $R$-module. Then the map

$$
\theta_{n}: A^{R}\left(y_{1}, \cdots, y_{n}\right) \longrightarrow A_{n}(V) \subseteq \operatorname{Hom}_{R}\left(C(V)^{\otimes n}, T(V)\right)
$$

given by $\theta_{n}\left(y_{j}\right)=y_{j}$ is a well-defined morphism of algebras.

Now we show that there exists a certain connected graded free $R$-module $V$ such that $\theta_{n}: A^{R}\left(y_{1}, \cdots, y_{n}\right) \longrightarrow A_{n}(V)$ is a monomorphism.

Lemma 3.6 Let $V$ be a free $R$-module with $\operatorname{dim}(V)=m$. Suppose that $m \geq n$. Then the homomorphism

$$
\theta_{n}: A^{R}\left(y_{1}, y_{2}, \cdots, y_{n}\right) \longrightarrow A_{n}(V)
$$

is a monomorphism.

Proof Let $X=\vee^{m} S^{2}$ be the wedge of $m$ copies of the 2-sphere $S^{2}$. Suppose that $m \geq n$. Let $V=H_{2}(X ; R)$. A basis for the $R$-algebra $A^{R}\left(y_{1}, \cdots, y_{n}\right)_{k}$ is given by

$$
y_{i_{\sigma(1)}} \cdots y_{i_{\sigma(k)}} \text {, }
$$

where $\left(i_{1}, \cdots, i_{k}\right)$ is taken over $1 \leq i_{1}<\cdots<i_{k} \leq n$ and $\sigma$ runs over all elements in $\Sigma_{k}$. Notice that $\theta_{n}\left(y_{i_{\sigma(1)}} \cdots y_{i_{\sigma(k)}}\right)$ is represented by the composite

$$
H_{*}\left(X^{n} ; R\right) \stackrel{p_{i_{\sigma(1)} \cdots i_{\sigma(k)}}}{\longrightarrow} H_{*}\left(X^{k} ; R\right) \stackrel{\gamma}{\longrightarrow} \widetilde{H}_{*}\left(X^{(k)} ; R\right)=V^{\otimes k} \longrightarrow T(V) .
$$

Let $\left\{x_{1}, \cdots, x_{m}\right\}$ be a basis for $V=H_{2}(X ; R)$. Let $1 \leq j_{1}<j_{2}<\cdots<j_{k} \leq n$ and let $z_{1}, \cdots, z_{n} \in C(V)$ be such that

(1) $z_{p}=1$ if $p \notin\left\{j_{1}, \cdots, j_{k}\right\}$;

(2) $z_{j_{s}}=x_{j_{s}}$. 
Then

$\theta_{n}\left(y_{i_{\sigma(1)}} \cdots y_{\left.i_{\sigma(k)}\right)}\right)\left(z_{1} \otimes z_{2} \otimes \cdots \otimes z_{n}\right)=\left\{\begin{array}{lr}0 & \text { for }\left(i_{1}, \cdots ; i_{k}\right) \neq\left(j_{1}, \cdots, j_{k}\right) \\ x_{j_{\sigma(1)}} \cdots x_{j_{\sigma(k)}} & \text { for }\left(i_{1}, \cdots, i_{k}\right)=\left(j_{1}, \cdots, j_{k}\right)\end{array}\right.$

The assertion follows.

Lemma 3.7 ((Lemma 2.1 in [6])) Let $\phi_{V}: V^{\otimes n} \longrightarrow V^{\otimes m}$ be a functorial $R$-linear map for any free $R$-module $V$ and let $x_{1}, \cdots, x_{n}$ be $n$ homogeneous elements in $V$.

(1) If $\operatorname{dim}_{R}(V)=n=m$, then the element $\phi_{V}\left(x_{1} \otimes \cdots \otimes x_{n}\right)$ belongs to the $R$-submodule of $V^{\otimes n}$ spanned by the elements

$$
x_{\sigma(1)} \otimes \cdots \otimes x_{\sigma(n)},
$$

where $\sigma$ runs through all elements in $\Sigma_{n}$.

(2) If $n \neq m$, then $\phi_{V}$ is the zero map.

Let $\operatorname{Hom}_{R}\left(C(-)^{\otimes n}, T(-)\right)$ be the set of all functorial $R$-linear maps from $C(V)^{\otimes n}$ to $T(V)$ with the convolution product. The same object we will be sometimes denoted by $\operatorname{Hom}_{R}^{\text {funct }}\left(C(V)^{\otimes n}, T(V)\right)$.

Proposition 3.8 The homomorphism

$$
\theta_{n}: A^{R}\left(y_{1}, \cdots, y_{n}\right) \longrightarrow \operatorname{Hom}_{R}\left(C(-)^{\otimes n}, T(-)\right)
$$

is an isomorphism of algebras.

Proof By Lemma 3.6, there is a free $R$-module $V$ such that

$$
\theta_{n}: A^{R}\left(y_{1}, \cdots, y_{n}\right) \longrightarrow A_{n}(V) \subseteq \operatorname{Hom}_{R}\left(C(V)^{\otimes n}, T(V)\right)
$$

is a monomorphism. Therefore from the following diagram

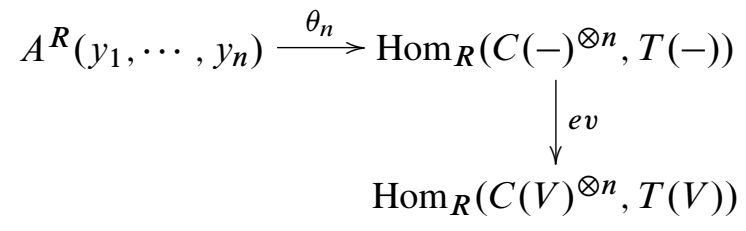

we have that the homomorphism

$$
\theta_{n}: A^{R}\left(y_{1}, \cdots, y_{n}\right) \longrightarrow \operatorname{Hom}_{R}\left(C(-)^{\otimes n}, T(-)\right)
$$

Algebraic $8 \mathcal{G}$ Geometric Topology, Volume 6 (2006) 
is a monomorphism. To show that $\theta_{n}$ is an epimorphism notice that for any $V$,

$$
\begin{gathered}
C(V)^{\otimes n}=(V \oplus R) \otimes \cdots \otimes(V \oplus R)=\bigoplus_{1 \leq i_{1}<\cdots<i_{t} \leq n} V^{\otimes t} \\
T(V)=\bigoplus_{m=0}^{\infty} V^{\otimes m} .
\end{gathered}
$$

and

By Lemma 3.7,

$$
\operatorname{Hom}_{R}\left(C(-)^{\otimes n}, T(-)\right)=\bigoplus_{1 \leq i_{1}<\cdots<i_{t} \leq n} \operatorname{Hom}_{R}^{\text {funct }}\left(V^{\otimes t}, V^{\otimes t}\right)=\bigoplus_{1 \leq i_{1}<\cdots<i_{t} \leq n} R\left(\Sigma_{t}\right)
$$

Now the assertion follows from Proposition 3.2.

Definition 3.9 The algebra $L_{n}^{R}$ is defined to be the equalizer of the homomorphisms

$$
\pi_{j}: A^{R}\left(y_{1}, \cdots, y_{n}\right) \longrightarrow A^{R}\left(y_{1}, \cdots, y_{n-1}\right)
$$

for $1 \leq j \leq n$, where the projection map $\pi_{j}$ is given by

$$
\pi_{j}\left(y_{k}\right)= \begin{cases}y_{k} & \text { for } k<j \\ 0 & \text { for } k=j \\ y_{k-1} & \text { for } k>j .\end{cases}
$$

By definition of $L_{n}^{R}$, as $\left.\pi_{i}\right|_{L_{n}^{R}}=\left.\pi_{j}\right|_{L_{n}^{R}}$ for $1 \leq i, j \leq n$, the homomorphisms $\pi_{j}: A^{R}\left(y_{1}, \cdots, y_{n}\right) \longrightarrow A^{R}\left(y_{1}, \cdots, y_{n-1}\right)$ induce a homomorphism $d_{n}: L_{n}^{R} \longrightarrow$ $L_{n-1}^{R}$ such that the diagram

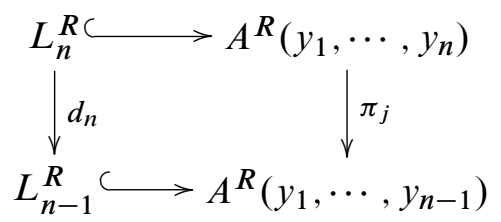

commutes for $1 \leq j \leq n$. The algebra $L_{\infty}^{R}$ is defined by the inverse limit

$$
L_{\infty}^{R}=\lim _{d_{n}} L_{n}^{R}
$$

The next objective is to find a connection between the combinatorial algebra $L_{n}^{R}$ for each $n \geq 0$ and related algebras of natural transformations of the tensor algebra $T(-)$. Consider the homomorphism

$$
\theta_{n}: A^{R}\left(y_{1}, \cdots, y_{n}\right) \longrightarrow A_{n}(V) \subseteq \operatorname{Hom}_{R}\left(C(V)^{\otimes n}, T(V)\right),
$$


of Lemma 3.4, where $V$ is a free $R$-module. Notice that $J_{n}(V)$ is the coequalizer of the homomorphisms

$$
\iota_{j}: C(V)^{\otimes(n-1)} \longrightarrow C(V)^{\otimes n}
$$

for $1 \leq j \leq n$, where $\iota_{j}$ is the composite

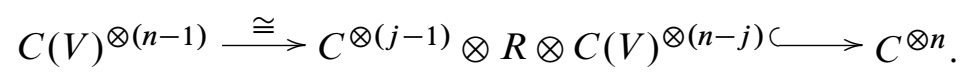

Let the $i$-th projection $p_{i}: C(V)^{\otimes n} \longrightarrow C(V)$ be given by

$$
p_{i}\left(x_{1} \otimes \cdots \otimes x_{n}\right)=\epsilon\left(x_{1}\right) \cdots \epsilon\left(x_{i-1}\right) x_{i} \epsilon\left(x_{i+1}\right) \cdots \epsilon\left(x_{n}\right) .
$$

Then

$$
p_{i} \circ \iota_{j}= \begin{cases}p_{i} & \text { for } i<j \\ \eta_{C(V)} \circ \epsilon_{C(V)^{\otimes(n-1)}} & \text { for } i=j \\ p_{i-1} & \text { for } i>j\end{cases}
$$

Thus there is a commutative diagram

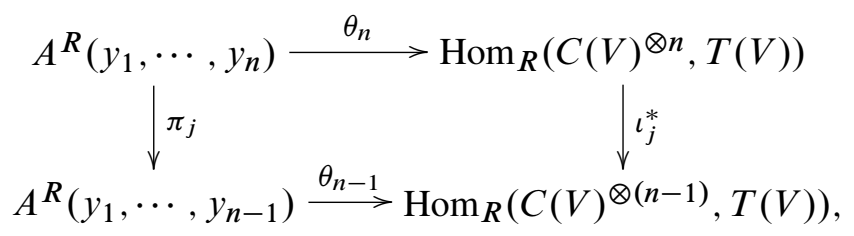

where $\pi_{j}$ is defined in Definition 3.9. Hence there exists a unique homomorphism of algebras

$$
\theta_{n}: L_{n}^{R} \longrightarrow \operatorname{Hom}_{R}\left(J_{n}(-), T(-)\right)
$$

such that the diagram

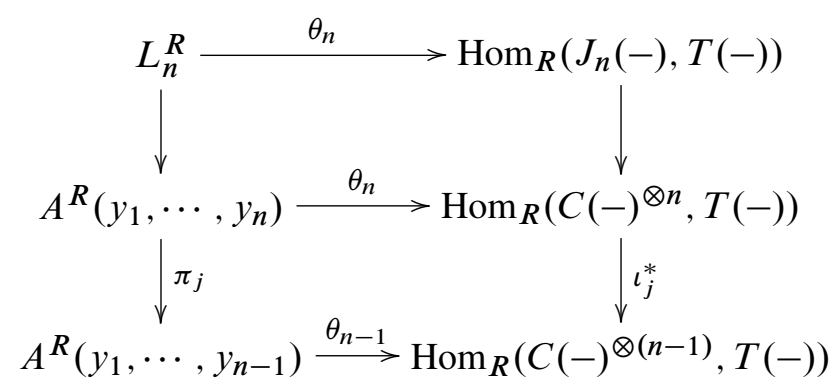

Algebraic $8 \mathcal{G}$ Geometric Topology, Volume 6 (2006) 
commutes for every $1 \leq j \leq n$. Furthermore, the homomorphism (3-1) preserves the projection homomorphisms, that is, there is a commutative diagram of algebras over $R$

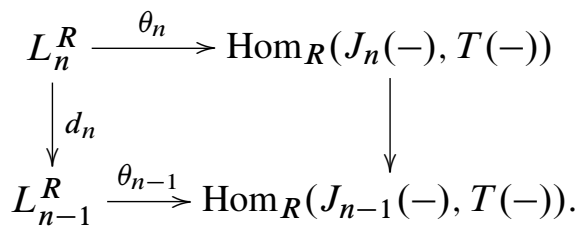

In the limit, this gives a homomorphism of co-filtered algebras

$$
\theta_{\infty}: L_{\infty}^{R} \longrightarrow \operatorname{Hom}_{R}(T(-), T(-))
$$

Theorem 3.10 The algebra $L_{\infty}^{R}$ is isomorphic to $\operatorname{Hom}_{R}(T(-), T(-))$ as cofiltered algebras, that is, the homomorphism

$$
\theta_{n}: L_{n}^{R} \longrightarrow \operatorname{Hom}_{R}\left(J_{n}(-), T(-)\right)
$$

is an isomorphism of algebras for each $n \geq 0$.

Proof The proof is given by induction on $n$. The assertion holds trivially in the cases $n=0,1$. Suppose that the assertion holds for $n-1$ with $n \geq 2$. By Lemma 3.6, the map

$$
\theta_{n}: A^{R}\left(y_{1}, \cdots, y_{n}\right) \longrightarrow \operatorname{Hom}_{R}\left(C(-)^{\otimes n}, T(-)\right)
$$

is a monomorphism. Thus the homomorphism

$$
\theta_{n}: L_{n}^{R} \longrightarrow \operatorname{Hom}_{R}\left(J_{n}(-), T(-)\right)
$$

is a monomorphism. Let $\Gamma_{n}$ be the kernel of the homomorphism

$$
L_{n}^{R} \longrightarrow L_{n-1}^{R}
$$

and let $\widetilde{\Gamma}_{n}$ be the kernel of the homomorphism

$$
\operatorname{Hom}_{R}\left(J_{n}(-), T(-)\right) \longrightarrow \operatorname{Hom}_{R}\left(J_{n-1}(-), T(-)\right) .
$$

Then

$$
\Gamma_{n}=\cap_{1 \leq j \leq n} \operatorname{ker}\left(\pi_{j}\right) \cong \Gamma_{n}\left(y_{1}, \cdots, y_{n}\right) \cong R\left(\Sigma_{n}\right),
$$

where $\Gamma_{n}\left(y_{1}, \cdots, y_{n}\right)$ is the R-submodule of $T_{n}\left(y_{1}, \cdots, y_{n}\right)$ spanned by the monomials $y_{\sigma(1)} \cdots y_{\sigma(n)}$ as $\sigma$ runs through all elements in $\Sigma_{n}$, and $R\left(\Sigma_{n}\right)$ is a group ring. Let $f \in \widetilde{\Gamma}_{n}$, that is, $f_{V}: J_{n}(V) \longrightarrow T(V)$ is such that $\left.f_{V}\right|_{J_{n-1}(V)}: J_{n-1}(V) \longrightarrow T(V)$ 
is zero. By assertion (2) of Lemma 3.7, there exists a natural map of R-modules $\phi_{V}: V^{\otimes n} \longrightarrow V^{\otimes n}$ such that the diagram

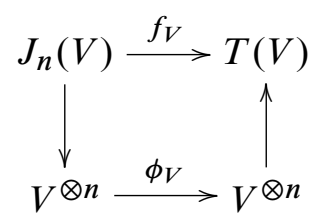

commutes for each $V$. By assertion (1) of Lemma 3.7, we have

$$
\phi_{V}\left(x_{1} \cdots x_{n}\right)=\sum_{\sigma \in \Sigma_{n}} k_{\sigma} x_{\sigma(1)} \cdots x_{\sigma(n)}
$$

for some coefficients $k_{\sigma} \in R$. Let

$$
z=\sum_{\sigma \in \Sigma_{n}} k_{\sigma} y_{\sigma(1)} \cdots y_{\sigma(n)} \in \gamma_{n} \subseteq L_{n}^{R} .
$$

Then it is routine to check that

$$
\theta_{n}(z)=f
$$

Thus the map

$$
\theta_{n}: \gamma_{n} \longrightarrow \tilde{\gamma}_{n}
$$

is an epimorphism. The assertion follows from the 5-Lemma.

By Lemma 3.6, we have

Corollary 3.11 Let $V$ be a free $R$-module with $\operatorname{dim}_{R} V \geq n$. Then the homomorphism

$$
\theta_{n}: L_{n}^{R} \longrightarrow \operatorname{Hom}_{R}\left(J_{n}(V), T(V)\right)
$$

is a monomorphism.

The algebra $L_{\infty}^{R}$ is called the universal convolution algebra.

Definition 3.12 The algebra $A_{n}^{R}[k]$ is defined as the quotient algebra of the tensor algebra generated by the words $\left\{y_{i_{1}}\left|y_{i_{2}}\right| \ldots \mid y_{i_{k}}\right\}$ with $1 \leq i_{j} \leq n$ for $1 \leq j \leq k$ over $R$ modulo the two sided ideal generated by the monomials

$$
\left\{y_{i_{1}}\left|y_{i_{2}}\right| \ldots \mid y_{i_{k}}\right\}\left\{y_{i_{k+1}}\left|y_{i_{k+2}}\right| \ldots \mid y_{i_{2 k}}\right\} \ldots\left\{y_{i_{(l-1) k+1}}\left|y_{i_{(l-1) k+2}}\right| \ldots \mid y_{i_{l k}}\right\}
$$

with $i_{s}=i_{t}$ for some $1 \leq s<t \leq k$. 
For two $R$-modules $C$ and $D$, define their smash product $C \wedge D$ to be the quotient module

$$
C \wedge D=(C \otimes D) /\left(C \otimes_{R} R \oplus R \otimes_{R} D\right) .
$$

Definition 3.13 Let $V$ be a free $R$-module. The algebra $A_{n}^{R}[k](V)$ is defined to be the subalgebra (under convolution) of $\operatorname{Hom}_{R}\left(C(V)^{\otimes n}, T\left(V^{\wedge k}\right)\right)$ generated by the elements $\left\{y_{i_{1}}\left|y_{i_{2}}\right| \ldots \mid y_{i_{k}}\right\}$ with $1 \leq i_{j} \leq n$ for $1 \leq j \leq k$ given by the composites

$$
\left\{y_{i_{1}}\left|y_{i_{2}}\right| \ldots \mid y_{i_{k}}\right\}: C(V)^{\otimes n} \stackrel{p_{i_{1}, \ldots, i_{k}}}{\longrightarrow} C(V)^{\otimes k} \stackrel{q}{\longrightarrow} V^{\wedge k} \stackrel{E}{\longrightarrow} T\left(V^{\wedge k}\right),
$$

where $p_{i_{1}, \ldots, i_{k}}: C(V)^{\otimes n} \longrightarrow C(V)$ is induced by

$$
p_{j}\left(x_{1} \otimes x_{2} \otimes \cdots \otimes x_{n}\right)=\epsilon\left(x_{1}\right) \cdots \epsilon\left(x_{j-1}\right) x_{j} \epsilon\left(x_{j+1}\right) \cdots \epsilon\left(x_{n}\right),
$$

$q: C(V) \longrightarrow V$ is the projection and $E: V^{\wedge k} \longrightarrow T\left(V^{\wedge k}\right)$ is the inclusion.

Lemma 3.14 Let $V$ be a connected graded free $R$-module. Then, in the algebra $A_{n}^{R}[k](V)$, the following equation holds for the generators $\left\{y_{i_{1}}\left|y_{i_{2}}\right| \ldots \mid y_{i_{k}}\right\}$ :

$$
\left\{y_{i_{1}}\left|y_{i_{2}}\right| \ldots \mid y_{i_{k}}\right\}\left\{y_{i_{k+1}}\left|y_{i_{k+2}}\right| \ldots \mid y_{i_{2 k}}\right\} \ldots\left\{y_{i_{(l-1) k+1}}\left|y_{i_{(l-1) k+2}}\right| \ldots \mid y_{i_{l k}}\right\}=0
$$

if $i_{j}=i_{k}$ for some $j \neq k$.

Proof The proof follows along the lines of the proof of Lemma 3.4.

Corollary 3.15 Let $V$ be a connected graded free $R$-module. Then the map

$$
\theta_{n}: A_{n}^{R}[k] \longrightarrow A_{n}^{R}[k](V) \subseteq \operatorname{Hom}_{R}\left(C(V)^{\otimes n}, T\left(V^{\wedge k}\right)\right)
$$

given by $\theta_{n}\left(\left\{y_{i_{1}}\left|y_{i_{2}}\right| \ldots \mid y_{i_{k}}\right\}\right)=\left\{y_{i_{1}}\left|y_{i_{2}}\right| \ldots \mid y_{i_{k}}\right\}$ is a well-defined morphism of algebras.

Lemma 3.16 Let $V$ be a free $R$-module with $\operatorname{dim}(V)=m$. Suppose that $m \geq n$. Then the homomorphism

$$
\theta_{n}: A_{n}^{R}[k] \longrightarrow A_{n}^{R}[k](V)
$$

is a monomorphism.

Proof The proof is analogous to that of Lemma 3.6, just with much clumsier notation. 
Proposition 3.17 The homomorphism

$$
\theta_{n}: A_{n}^{R}[k] \longrightarrow \operatorname{Hom}_{R}\left(C(-)^{\otimes n}, T\left(-^{\wedge k}\right)\right)
$$

is an isomorphism of algebras.

Proof By Lemma 3.16, the homomorphism $\theta_{n}: A_{n}^{R}[k] \longrightarrow \operatorname{Hom}_{R}\left(C(V)^{\otimes n}\right.$, $\left.T\left(V^{\wedge k}\right)\right)$ is monomorphism for a certain choice of a free $R$-module $V$. To prove that $\theta$ is an epimorphism, notice that

$$
C(V)^{\otimes n}=(V \oplus R) \otimes \cdots \otimes(V \oplus R)=\bigoplus_{1 \leq i_{1}<\cdots<i_{t} \leq n}(V)^{\otimes t}
$$

Now, the assertion follows by looking at the homomorphism $\theta$ in each degree and applying Lemma 3.7.

Notice that the homomorphisms

$$
\pi_{j+\{1, \ldots, l\}}: A_{l n}^{R}\left(x_{1}, x_{2}, \ldots, x_{l n}\right) \longrightarrow A_{l(n-1)}^{R}\left(x_{1}, x_{2}, \ldots, x_{l(n-1)}\right)
$$

given by

$$
\pi_{j+\{1, \ldots, l\}}\left(x_{i}\right)= \begin{cases}x_{i} & \text { for } i<j+1 \\ 1 & \text { for } j+1 \leq i \leq j+l \\ x_{i-1} & \text { for } i>j+l\end{cases}
$$

for $0 \leq j \leq n-1$ induce homomorphisms

$$
\pi_{j+\{1, \ldots, l\}}: A_{l n}^{R}[k]\left(x_{1}, x_{2}, \ldots, x_{l n}\right) \longrightarrow A_{l(n-1)}^{R}[k]\left(x_{1}, x_{2}, \ldots, x_{l(n-1)}\right) .
$$

Definition 3.18 The algebra ${ }^{R} L_{n}^{(l)(k)}$ is defined to be the subgroup of $A_{l n}^{R}[k]$ given by the equalizer of the projections

$$
\begin{gathered}
A_{l n}^{R}[k] \cap\left(\bigcap_{j=0}^{n-1}\left(\bigcap_{i=1}^{l} \operatorname{Ker} \pi_{j l+i}\right)\right) \\
\pi_{j+\{1, \ldots, l\}}|\cdot| \\
A_{l(n-1)}^{R}[k] \cap\left(\bigcap_{j=0}^{n-2}\left(\bigcap_{i=1}^{l} \operatorname{Ker} \pi_{j l+i}\right)\right)
\end{gathered}
$$

for $0 \leq j \leq n-1$. 
As ${ }^{R} L_{n}^{(l)(k)}$ is the equalizer of the projections $\pi_{j+\{1, \ldots, l\}}: A_{l n}^{R}[k] \longrightarrow A_{l(n-1)}^{R}[k]$, they induce a projective homomorphism $d_{n}:{ }^{R} L_{n}^{(l)(k)} \longrightarrow{ }^{R} L_{n-1}^{(l)(k)}$ such that the diagram

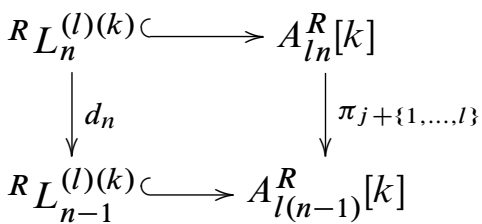

commutes for each $1 \leq j \leq n-1$. The algebra ${ }^{R} L_{\infty}^{(l)(k)}$ is defined by the inverse limit

$$
{ }^{R} L_{\infty}^{(l)(k)}=\lim _{d_{n}}{ }^{R} L_{n}^{(l)(k)}
$$

The following theorem is the analogue of Theorem 3.10.

Theorem 3.19 The algebra ${ }^{R} L_{\infty}^{(l)(k)}$ is isomorphic to $\operatorname{Hom}_{R}\left(T\left(-^{\wedge}\right), T\left(-^{\wedge k}\right)\right)$ as cofiltered algebras, that is, the homomorphism

$$
\theta_{n}:{ }^{R} L_{n}^{(l)(k)} \longrightarrow \operatorname{Hom}_{R}\left(J_{n}\left(-^{\wedge l}\right), T\left(-^{\wedge k}\right)\right)
$$

is an isomorphism of algebras for each $n \geq 0$.

Proof For each $n \geq 1$, there is a commutative diagram

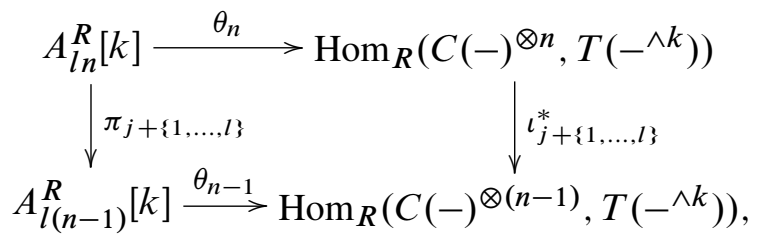

where $\pi_{j+\{1, \ldots, l\}}$ is induced by projections (3-2). Hence there exists a unique homomorphism of algebras

$$
\theta_{n}:{ }^{R} L_{n}^{(l)(k)} \longrightarrow \operatorname{Hom}_{R}\left(J_{n}\left(-^{\wedge l}\right), T\left(-^{\wedge k}\right)\right)
$$

Algebraic $\mathcal{B}$ Geometric Topology, Volume 6 (2006) 
such that the diagram

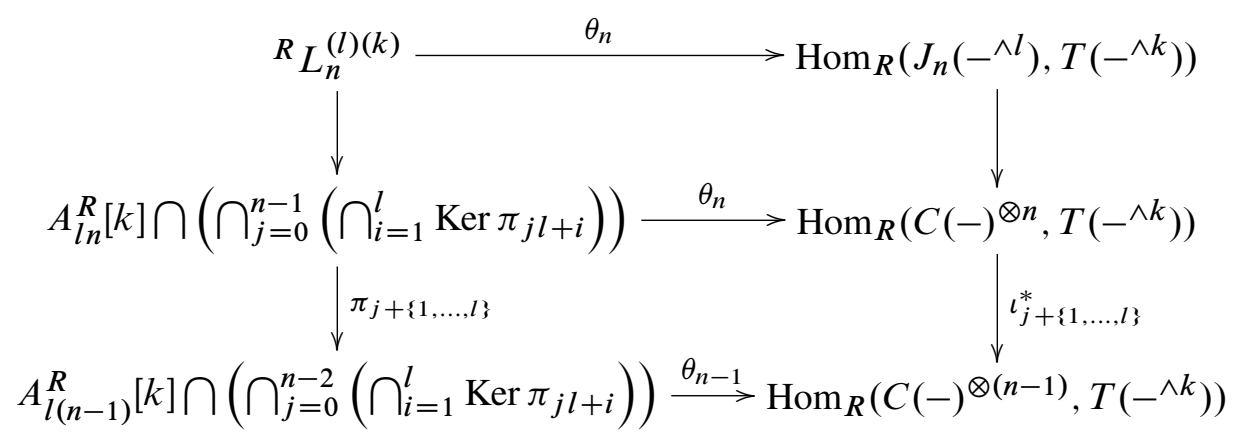

commutes for every $0 \leq j \leq n-1$.

As the homomorphisms $\theta_{n}$ and $\theta_{n-1}$ in the bottom two rows are isomorphisms of algebras, it follows that $\theta_{n}:{ }^{R} L_{n}^{(l)(k)} \longrightarrow \operatorname{Hom}_{R}\left(J_{n}\left(-^{\wedge l}\right), T\left(-^{\wedge k}\right)\right)$ is an isomorphism of algebras as well.

Furthermore, there is a commutative diagram of algebras over $R$

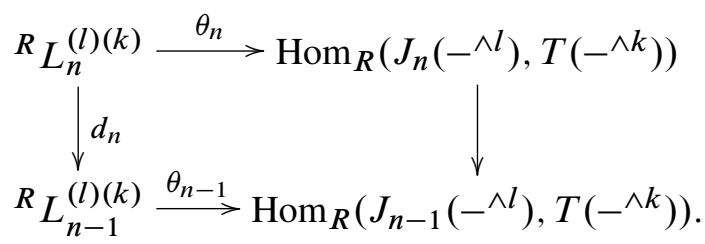

This gives an isomorphism of co-filtered algebras

$$
\theta_{\infty}:{ }^{R} L_{\infty}^{(l)(k)} \longrightarrow \operatorname{Hom}_{R}\left(T\left(-^{\wedge l}\right), T\left(-^{\wedge k}\right)\right) .
$$

The assertion follows.

Remark In the case when $k=1$ we will drop the superscript $k$ in the above constructions. For example, ${ }^{R} L_{n}^{(l)(1)}$ will be denoted by ${ }^{R} L_{n}^{(l)}$, and the algebra $\operatorname{Hom}_{R}\left(J_{n}\left(-^{\wedge l}\right), T\left(-^{\wedge 1}\right)\right)$ will be denoted by $\operatorname{Hom}_{R}\left(J_{n}\left(-^{\wedge l}\right), T(-)\right)$.

\section{Combinatorial groups and natural coalgebra transforma- tions of tensor algebras}

In this section a relation between combinatorial groups and natural coalgebra transformations of tensor algebras is established. As a consequence, there are representations 
of the combinatorial groups into algebras of natural linear transformations of tensor algebras.

In the algebra $A^{R}\left(y_{1}, \cdots, y_{n}\right)$, since $y_{j}^{2}=0$, we have $\left(1+r y_{j}\right)\left(1-r y_{j}\right)=1$ for $r \in R$ and $1 \leq j \leq n$. Thus the element $1+r y_{j}$ is a unit element for $r \in R$ and $1 \leq j \leq n$.

Definition 4.1 Let $R$ be a commutative ring with identity. Define the group $G\left(A^{R}\left(y_{1}, \cdots, y_{n}\right)\right)$ to be the subgroup of the group of units in $A^{R}\left(y_{1}, \cdots, y_{n}\right)$ generated by the elements $1+r y_{j}$ for $r \in R$ and $1 \leq j \leq n$.

Remark Cohen [1] proved that if $R=\mathbb{Z}$ or $\mathbb{Z} / p^{r}$, then $G\left(A^{R}\left(y_{1}, \cdots, y_{n}\right)\right)$ is isomorphic to the group $K_{n}^{R}$.

Let $G$ be a group and let $x, y \in G$. We write $[x, y]$ for the commutator $x^{-1} y^{-1} x y$. The notation $\left[\left[x_{1}, x_{2}, \cdots, x_{t}\right]\right.$ represents the iterated commutator from left to right, that is $\left[\left[x_{1}, x_{2}, \cdots, x_{t}\right]=\left[\left[x_{1}, x_{2}\right], \cdots, x_{t}\right]\right.$.

Lemma 4.2 Let $1 \leq j_{1}, \cdots, j_{t} \leq n$ and let $r_{s} \in R$ for $1 \leq s \leq t$. Let $x_{s}=1+r_{s} y_{j_{s}} \in$ $G\left(A^{R}\left(y_{1}, \cdots, y_{n}\right)\right)$. Then

(1) $\left[\left[x_{1}, x_{2}, \cdots, x_{t}\right]=1+r_{1} r_{2} \cdots r_{t}\left[\left[y_{j_{1}}, y_{j_{2}}, \cdots, y_{j_{t}}\right]\right.\right.$;

(2) Let $m=m_{1} \cdot m_{2} \cdots m_{t}$. Then $\left[\left[x_{1}^{m_{1}}, x_{2}^{m_{2}}, \cdots, x_{t}^{m_{t}}\right]=1+m r_{1} r_{2} \cdots r_{t}\left[\left[y_{j_{1}}, y_{j_{2}}, \cdots, y_{j_{t}}\right]\right.\right.$

where $\left[\left[x_{1}, x_{2}, \cdots, x_{t}\right]\right.$ is the iterated commutator in the group $G\left(A^{R}\left(y_{1}, \cdots, y_{n}\right)\right)$ and $\left[\left[y_{j_{1}}, y_{j_{2}}, \cdots, y_{j_{t}}\right]\right.$ is the iterated commutator in the algebra $A^{R}\left(y_{1}, \cdots, y_{n}\right)$.

Proof The proof of part (1) is given by induction on $t$. The assertion holds obviously for $t=1$. Suppose that the assertion holds for $t-1$. Then $\left[\left[x_{1}, x_{2}, \cdots, x_{t-1}\right]=1+\right.$ $r_{1} r_{2} \cdots r_{t-1}\left[\left[y_{j_{1}}, y_{j_{2}}, \cdots, y_{j_{t-1}}\right]\right.$. Notice that

$$
\left(1+r_{1} r_{2} \cdots r_{t-1}\left[\left[y_{j_{1}}, y_{j_{2}}, \cdots, y_{j_{t-1}}\right]\right)\left(1-r_{1} r_{2} \cdots r_{t-1}\left[\left[y_{j_{1}}, y_{j_{2}}, \cdots, y_{j_{t-1}}\right]\right)=1 .\right.\right.
$$

Thus $\left[\left[x_{1}, x_{2}, \cdots, x_{t-1}\right]^{-1}=1-r_{1} r_{2} \cdots r_{t-1}\left[\left[y_{j_{1}}, y_{j_{2}}, \cdots, y_{j_{t-1}}\right]\right.\right.$ and so

$$
\begin{gathered}
{\left[\left[x_{1}, x_{2}, \cdots, x_{t}\right]=\left[[ x _ { 1 } , x _ { 2 } , \cdots , x _ { t - 1 } ] ^ { - 1 } x _ { t } ^ { - 1 } \left[\left[x_{1}, x_{2}, \cdots, x_{t-1}\right] x_{t}\right.\right.\right.} \\
=\left(1-r_{1} r_{2} \cdots r_{t-1} \alpha\right)\left(1-r_{t} y_{j_{t}}\right)\left(1+r_{1} r_{2} \cdots r_{t-1} \alpha\right)\left(1+r_{t} y_{j_{t}}\right) \\
=1+r_{1} r_{2} \cdots r_{t}\left[\left[y_{j_{1}}, y_{j_{2}}, \cdots, y_{j_{t}}\right]\right.
\end{gathered}
$$

where $\alpha=\left[\left[y_{j_{1}}, y_{j_{2}}, \cdots, y_{j_{t-1}}\right]\right.$. The induction is finished and the assertion of part (1) follows.

The proof of part (2) is similar calculation to that in part (1) and is done by induction on $t$. 
Proposition 4.3 For any commutative ring with identity, there is a group isomorphism

$$
\exp : K_{n}^{R} \longrightarrow G\left(A^{R}\left(y_{1}, \cdots, y_{n}\right)\right)
$$

given by

$$
\exp \left(x_{i}^{r}\right)=1+r y_{i} \text {. }
$$

Proof As the proof is similar to the proof given by Cohen [1] in the case $R=\mathbb{Z}$ or $\mathbb{Z} / p^{r}$, we just outline the general idea. By inspection of Lemma 4.2, the exponent map preserves the defining relations of $K_{n}^{R}$ and thus induces a group homomorphism. It is by definition obviously an epimorphism. That exp is a monomorphism follows by induction on the descending central series filtration of $K_{n}^{R}$ and noticing that $K_{n}^{R}$ is a nilpotent group of class $n$.

Let $\bar{V}=\left\langle x_{1}, \cdots, x_{n}\right\rangle$ be the free $R$-module generated by $x_{1}, \cdots, x_{n}$. Let $\gamma_{n}^{R}$ be the $R$-submodule of $\bar{V}^{\otimes n}$ generated by the homogenous elements $x_{\sigma(1)} x_{\sigma(2)} \cdots x_{\sigma(n)}$ for $\sigma \in S_{n}$. Let Lie ${ }^{R}(n)$ be the $R$-submodule of $\gamma_{n}^{R}$ generated by the $n$-fold commutators $\left[\left[x_{\sigma(1)}, x_{\sigma(2)}, \cdots x_{\sigma(n)}\right]\right.$ for $\sigma \in S_{n}$. Let $P_{n}(T(V))$ be the set of primitive elements of $T(V)$ of tensor length $n$.

Lemma 4.4 $\gamma_{n}^{R} \cap P_{n}(T(\bar{V}))=\operatorname{Lie}^{R}(n)$.

Proof If $R=\mathbb{Z}$, then $P(T(\bar{V}))=L(\bar{V})$ is the free Lie algebra generated by $\bar{V}$. Thus $\gamma_{n}^{\mathbb{Z}} \cap P_{n}(T(\bar{V}))=\gamma_{n}^{\mathbb{Z}} \cap L_{n}(\bar{V})=\operatorname{Lie}^{\mathbb{Z}}(n)$.

Let $p$ be a prime. If $R=\mathbb{Z} / p$, then $P(T(\bar{V}))=L^{\text {res }}(\bar{V})$ is the free restricted Lie algebra generated by $V$. Selick and Wu [6] proved that $\gamma_{n}^{\mathbb{Z} / p} \cap L_{n}^{\text {res }}(\bar{V}) \subseteq L_{n}(\bar{V})$. Thus

$$
\gamma_{n}^{\mathbb{Z} / p} \cap P_{n}(T(\bar{V}))=\gamma_{n}^{\mathbb{Z} / p} \cap L_{n}^{\mathrm{res}}(\bar{V})=\gamma_{n}^{\mathbb{Z} / p} \cap L_{n}(\bar{V})=\operatorname{Lie}^{\mathbb{Z} / p}(n) .
$$

Now assume $R$ is a commutative ring with identity. Let

$$
\bar{\psi}: T(\bar{V}) \longrightarrow T(\bar{V}) \otimes T(\bar{V})
$$

be the reduced comultiplication, that is, $\bar{\psi}(x)=\psi(x)-x \otimes 1-1 \otimes x$. Then $P(T(V))$ is the kernel of $\bar{\psi}: T(\bar{V}) \longrightarrow T(\bar{V}) \otimes T(\bar{V})$. Let $\psi_{s}: V^{\otimes n} \longrightarrow V^{\otimes s} \otimes V^{\otimes n-s}$ be defined by

$$
\psi\left(a_{1} a_{2} \cdots a_{n}\right)=\sum_{(I, J)} a_{i_{1}} a_{i_{2}} \cdots a_{i_{s}} \otimes a_{j_{1}} a_{j_{2}} \cdots a_{j_{n-s}},
$$

where $I=\left(i_{1}, i_{2}, \cdots, i_{s}\right)$ with $i_{1}<i_{2}<\cdots<i_{s}, J=\left(j_{1}, j_{2}, \cdots, j_{n-s}\right)$ with $j_{1}<j_{2}<\cdots<j_{n-s}$ and $(I, J)$ runs over all $(s, n-s)$-shuffles. Then $\left.\bar{\psi}\right|_{V \otimes n}=$ $\sum_{s=1}^{n-1} \psi_{s}: V^{\otimes n} \longrightarrow \bigoplus_{s=1}^{n-1} V^{\otimes s} \otimes V^{\otimes n-s} \subseteq T(V) \otimes T(V)$ for any $V$ and so $\gamma_{n}^{R} \cap$ 
$P_{n}(T(\bar{V}))$ is the kernel of the map $\left.\bar{\psi}\right|_{\gamma_{n}^{R}}=\left.\sum_{s=1}^{n-1} \psi_{s}\right|_{\gamma_{n}^{R}}: \gamma_{n}^{R} \longrightarrow \bigoplus_{s=1}^{n-1} \bar{V}^{\otimes s} \otimes$ $\bar{V}^{\otimes n-s}$. Let $\bar{V}^{\prime}$ be the free $\mathbb{Z}$-module generated by $x_{1}, x_{2}, \cdots, x_{n}$. Then

$\left.\bar{\psi}\right|_{\gamma_{n}^{R}}=\left.\bar{\psi}\right|_{\gamma_{n}^{\mathbb{Z}}} \otimes_{\mathbb{Z}} R: \gamma_{n}^{R}=\gamma_{n}^{\mathbb{Z}} \otimes_{\mathbb{Z}} R \rightarrow \bigoplus_{s=1}^{n-1} \bar{V}^{\otimes s} \otimes \bar{V}^{\otimes n-s}=\bigoplus_{s=1}^{n-1} \bar{V}^{\prime \otimes s} \otimes_{\mathbb{Z}} \bar{V}^{\prime \otimes n-s} \otimes_{\mathbb{Z}} R$.

Notice that $\operatorname{Ker}\left(\left.\bar{\psi}\right|_{\gamma_{n}^{\mathbb{Z}}}\right)=\operatorname{Lie}^{\mathbb{Z}}(n)$. Thus $\left.\bar{\psi}\right|_{\gamma_{n}^{\mathbb{Z}}}$ factors through $\gamma_{n}^{\mathbb{Z}} / \operatorname{Lie}^{\mathbb{Z}}(n)$ and the resulting map $j: \gamma_{n}^{\mathbb{Z}} / \operatorname{Lie}^{\mathbb{Z}}(n) \longrightarrow \bigoplus_{s=1}^{n-1} \bar{V}^{\prime} \otimes^{s} \otimes_{\mathbb{Z}} \bar{V}^{\prime} \otimes^{n-s}$ is a monomorphism. Notice that $\operatorname{Ker}\left(\left.\bar{\psi}\right|_{\gamma_{n}^{\mathbb{Z} / p}}\right)=\operatorname{Lie}^{\mathbb{Z} / p}(n)$ for any prime $p$. Thus

$$
\begin{aligned}
& j \otimes_{\mathbb{Z}} \mathbb{Z} / p:\left(\gamma_{n}^{\mathbb{Z}} / \operatorname{Lie}^{\mathbb{Z}}(n)\right) \otimes_{\mathbb{Z}} \mathbb{Z} / p=\gamma_{n}^{\mathbb{Z} / p} / \operatorname{Lie}^{\mathbb{Z} / p}(n) \\
& \longrightarrow \bigoplus_{s=1}^{n-1} \bar{V}^{\prime \otimes s} \otimes_{\mathbb{Z}} \bar{V}^{, \otimes n-s} \otimes_{\mathbb{Z}} \mathbb{Z} / p
\end{aligned}
$$

is a monomorphism for any prime $p$ and so the cokernel of

$$
j: \gamma_{n}^{\mathbb{Z}} / \operatorname{Lie}^{\mathbb{Z}}(n) \longrightarrow \bigoplus_{s=1}^{n-1} \bar{V}^{\prime \otimes s} \otimes_{\mathbb{Z}} \bar{V}^{\prime \otimes n-s}
$$

is a torsion free finitely generated $\mathbb{Z}$-module. Thus

$$
j \otimes_{\mathbb{Z}} R:\left(\gamma_{n}^{\mathbb{Z}} / \operatorname{Lie}^{\mathbb{Z}}(n)\right) \otimes_{\mathbb{Z}} R \longrightarrow \bigoplus_{s=1}^{n-1} \bar{V}^{\prime} \otimes s \otimes_{\mathbb{Z}} \bar{V}^{\prime \otimes n-s} \otimes_{\mathbb{Z}} R
$$

is a monomorphism for any ring $R$ and so the image of

$$
\left.\bar{\psi}\right|_{\gamma_{n}^{R}}: \gamma_{n}^{R} \longrightarrow \bigoplus_{s=1}^{n-1} \bar{V}^{\otimes s} \otimes_{R} \bar{V}^{\otimes n-s}
$$

is isomorphic to $\left(\gamma_{n}^{\mathbb{Z}} / \operatorname{Lie}^{\mathbb{Z}}(n)\right) \otimes_{\mathbb{Z}} R$. Notice that $\gamma_{n}^{\mathbb{Z}} / \operatorname{Lie}^{\mathbb{Z}}(n)$ is a free $\mathbb{Z}$-module. From the short exact sequence

$$
\operatorname{Lie}^{\mathbb{Z}}(n) \smile \gamma_{n}^{\mathbb{Z}} \longrightarrow \gamma_{n}^{\mathbb{Z}} / \operatorname{Lie}^{\mathbb{Z}}(n),
$$

we get the short exact sequence

$$
\operatorname{Lie}^{R}(n)=\operatorname{Lie}^{\mathbb{Z}}(n) \otimes_{\mathbb{Z}} R^{\longrightarrow} \gamma_{n}^{R}=\gamma_{n}^{\mathbb{Z}} \otimes_{\mathbb{Z}} R \longrightarrow \gamma_{n}^{\mathbb{Z}} / \operatorname{Lie}^{\mathbb{Z}}(n) \otimes_{\mathbb{Z}} R .
$$

The assertion follows.

Algebraic $\&$ Geometric Topology, Volume 6 (2006) 


\section{The group of natural coalgebra transformations of $\left\{J_{n}(-)\right\}_{n \geq 0}$}

Now we determine the set of functorial coalgebra maps from $C(V)^{\otimes n}$ to $T(V)$. Let Coalg $\left(C(-)^{\otimes n}, T(-)\right)$ be the set of all functorial maps of coalgebras from $C(V)^{\otimes n}$ to $T(V)$, with the multiplication given by the convolution product. Notice that Coalg $\left(C(-)^{\otimes n}, T(-)\right)$ is a group with the convolution product.

Theorem 4.5 There is an isomorphism of groups

$$
e: K_{n}^{R} \longrightarrow \operatorname{Coalg}\left(C(-)^{\otimes n}, T(-)\right) \quad \text { for each } n \geq 0 .
$$

Proof Let $\theta_{n}: A^{R}\left(y_{1}, \cdots, y_{n}\right) \longrightarrow \operatorname{Hom}_{R}\left(C(-)^{\otimes n}, T(-)\right)$ be the algebra homomorphism in Proposition 3.8. Notice that

$$
\theta_{n}\left(1+r y_{j}\right): C(V)^{\otimes n} \longrightarrow T(V)
$$

is a functorial map of coalgebras for $r \in R$ and $1 \leq j \leq n$. Thus

$$
\theta_{n}\left(G\left(A^{R}\left(y_{1}, \cdots, y_{n}\right)\right)\right) \subseteq \operatorname{Coalg}\left(C(-)^{\otimes n}, T(-)\right)
$$

and so

$$
e=\left.\theta_{n}\right|_{G\left(A^{R}\left(y_{1}, \cdots, y_{n}\right)\right)}: G\left(A^{R}\left(y_{1}, \cdots, y_{n}\right)\right) \longrightarrow \operatorname{Coalg}\left(C(-)^{\otimes n}, T(-)\right)
$$

is a well-defined monomorphism of groups. We need to show that $e$ is an epimorphism. By Proposition 4.3, we can identify $K_{n}^{R}$ with $G\left(A^{R}\left(y_{1}, \cdots, y_{n}\right)\right)$.

By assuming that $V$ is a connected module, $C(V)$ is a connected graded coalgebra. Let $\mathrm{sk}_{t} C(V)^{\otimes n}=\bigoplus_{j \leq 2 t}\left(C(V)^{\otimes n}\right)_{j}$. Then $\left\{\mathrm{sk}_{t} C(V)^{\otimes n}\right\}$ is a (finite) coalgebra filtration of $C(V)^{\otimes n}$. Let $\left\{\Gamma^{t} K_{n}^{R}\right\}$ be the descending central series of $K_{n}^{R}$ starting with $\Gamma^{1} K_{n}^{R}=K_{n}^{R}$. Notice that by Lemma 4.2,

$$
\Gamma^{t} K_{n}^{R} \subseteq\{1\}+I^{t} A^{R}\left(y_{1}, y_{2}, \cdots, y_{n}\right)
$$

for each $t \geq 1$, where $I^{t} A^{R}\left(y_{1}, y_{2}, \cdots, y_{n}\right)$ is the $t$-fold product of the augmentation ideal $I A^{R}\left(y_{1}, y_{2}, \cdots, y_{n}\right)$. Let $\mathrm{sk}_{t}$ denote $\mathrm{sk}_{t} C(-)^{\otimes n}$. Then the composite

$$
\Gamma^{s} K_{n}^{R C} \longrightarrow K_{n}^{R} \stackrel{e}{\longrightarrow} \operatorname{Coalg}\left(C(-)^{\otimes n}, T(-)\right) \longrightarrow \operatorname{Coalg}\left(\mathrm{sk}_{t}, T(-)\right)
$$

is the trivial map if $s>t$ and so there is a commutative diagram

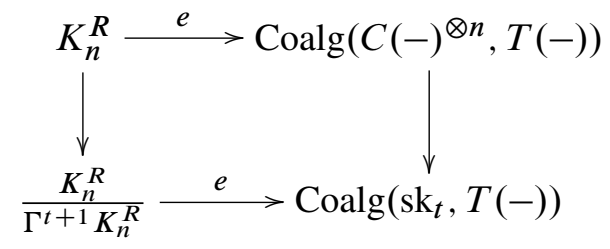

Algebraic 83 Geometric Topology, Volume 6 (2006) 
for each $t \geq 1$.

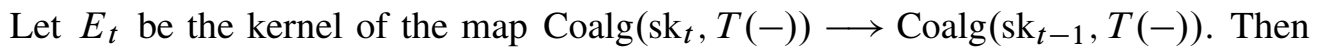
there is a commutative diagram

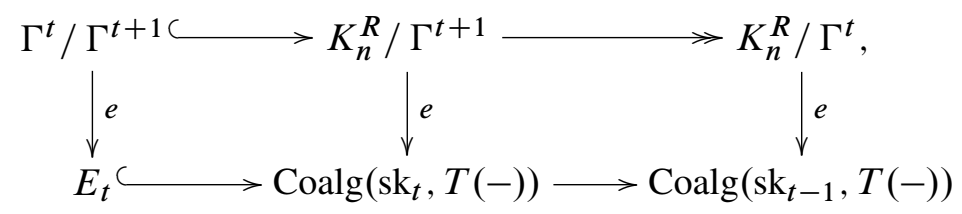

where $\Gamma^{t}=\Gamma^{t} K_{n}^{R}$. Notice that

$$
\Gamma^{n+1}=E_{n+1}=\{1\} \quad \text { and } \quad K_{n}^{R} / \Gamma^{1}=\operatorname{Coalg}\left(\mathrm{sk}_{0}, T(-)\right)=\{1\} .
$$

It suffices to show that $e: \Gamma^{t} / \Gamma^{t+1} \longrightarrow E_{t}$ is an epimorphism for each $t \geq 1$.

Notice that $f \in E_{t} \subseteq \operatorname{Hom}_{R}\left(\mathrm{sk}_{t}, T(-)\right)$ if and only if $f: \mathrm{sk}_{t} C(V)^{\otimes n} \longrightarrow T(V)$ is a functorial $R$-linear map such that

(1) $\left.f\right|_{\left(C(V)^{\otimes n}\right)_{j}}=0:\left(C(V)^{\otimes n}\right)_{j} \longrightarrow T(V)_{j}$ for $0<j<2 t$;

(2) $f\left(\left(C(V)^{\otimes n}\right)_{2 t}\right) \subseteq P\left(T(V)_{2 t}\right)=P_{t}(T(V))$.

Now let $f \in E_{t}$ and let $\tilde{f}: C(V)^{\otimes n} \longrightarrow T(V)$ be the functorial $R$-linear map such that $\left.\tilde{f}\right|_{\mathrm{sk}_{t}}=f$ and $\left.\tilde{f}\right|_{\left(C(V)^{\otimes n}\right)_{j}}=0$ for $j>2 t$. Let $1 \leq i_{1}<i_{2}<\cdots<i_{t} \leq n$ be a sequence of length $t$. Let

$$
\lambda_{I}: C(V)^{\otimes t}=R \otimes \cdots R \otimes \stackrel{\left(i_{1}\right)}{C}(V) \otimes R \cdots \otimes R \otimes C^{\left(i_{t}\right)}(V) \otimes R \cdots \otimes R \longrightarrow C(V)^{\otimes n}
$$

be the inclusion of $i_{1}, i_{2}, \cdots, i_{t}$ coordinates and let

$$
\pi_{I}=\epsilon \otimes \cdots \otimes \operatorname{id}_{C(V)}^{\left(i_{1}\right)} \otimes \cdots \otimes \operatorname{id}_{C(V)}^{\left(i_{t}\right)} \otimes \cdots \otimes \epsilon: C(V)^{\otimes n} \longrightarrow C(V)^{\otimes t}
$$

be the projection of $i_{1}, i_{2}, \cdots, i_{t}$ coordinates. Let $g_{I}: C(V)^{\otimes t} \longrightarrow T(V)$ be the composite

$$
C(V)^{\otimes t} \stackrel{\lambda_{I}}{\longrightarrow} C(V)^{\otimes n} \stackrel{\tilde{f}}{\longrightarrow} T(V)
$$

and let $\tilde{f}_{I}=g_{I} \circ \pi_{I}: C(V)^{\otimes n} \longrightarrow T(V)$. Notice that

$$
\tilde{f}_{I}\left(\left(C(V)^{\otimes n}\right)_{2 t}\right)=f_{I}\left(\left(C(V)^{\otimes n}\right)_{2 t}\right) \subseteq P(T(V)) .
$$

Thus $g_{I}\left(\left(C(V)^{\otimes t}\right)_{2 t}\right)=g_{I}\left(V^{\otimes t}\right) \subseteq P(T(V))$. Let $\bar{V}, \gamma_{t}^{R}$ and $\operatorname{Lie}^{R}(t)$ be defined as in Lemma 4.4. By Lemmas 3.7 and 4.4, we have

$$
g_{I}\left(\gamma_{t}^{R}\right) \subseteq \gamma_{t}^{R} \cap P_{t}(\bar{V})=\operatorname{Lie}^{R}(n)
$$

Algebraic 83 Geometric Topology, Volume 6 (2006) 
Thus

$$
g_{I}\left(x_{1} x_{2} \cdots x_{t}\right)=\sum_{\tau \in S_{n-1}} r_{\tau}\left[\left[x_{1}, x_{\tau(2)}, \cdots, x_{\tau(t)}\right]\right.
$$

for some $r_{\tau} \in R$ for each $\tau \in \Sigma_{n-1}$, where $\Sigma_{n-1}$ acts on $\{2,3, \cdots, n\}$. Notice that $g_{I}$ is a functorial map. Thus

$$
g_{I}\left(a_{1} a_{2} \cdots a_{t}\right)=\sum_{\tau \in S_{n-1}} r_{\tau}\left[\left[a_{1}, a_{\tau(2)}, \cdots, a_{\tau(t)}\right]\right.
$$

for any free $R$-module $V$ and any $a_{j} \in V$. Let

$$
w_{I}=\prod_{\tau \in S_{n-1}}\left(1+r_{\tau}\left[\left[y_{i_{1}}, y_{i_{\tau(2)}}, \cdots, y_{i_{\tau(t)}}\right]\right) \in A^{R}\left(y_{1}, y_{2}, \cdots, y_{n}\right)\right.
$$

where the product is taken in an arbitrary order. Then it is routine to check that

$$
\left.\theta_{n}\left(w_{I}\right)\right|_{\mathrm{sk}_{t}}=\left.g_{I} \circ \pi_{I}\right|_{\mathrm{sk}_{t}}=\left.\tilde{f}_{I}\right|_{\mathrm{sk}_{t}}: \mathrm{sk}_{t} C(V)^{\otimes n} \longrightarrow T(V) .
$$

By Lemma $4.2, w_{I} \in K_{n}^{R}$ and so

$$
\left.\tilde{f}_{I}\right|_{\mathrm{sk}_{t}} \in \operatorname{Im}\left(e: \Gamma^{t} / \Gamma^{t+1} \longrightarrow E_{t}\right) .
$$

Notice that

$$
\left(C(V)^{\otimes n}\right)_{2 t}=\bigoplus_{1 \leq i_{1}<i_{2}<\cdots<i_{t} \leq n} V^{\otimes t}=\bigoplus_{\left(\begin{array}{c}
n \\
t
\end{array}\right)} V^{\otimes t}
$$

Thus

$$
f=\left.\tilde{f}\right|_{\mathrm{sk}_{t}}=\left.\prod_{I} \tilde{f}_{I}\right|_{\mathrm{sk}_{t}}
$$

is in the convolution algebra $\operatorname{Hom}_{R}\left(\mathrm{sk}_{t} C(-)^{\otimes n}, T(-)\right)$, where $I=\left(i_{1}, i_{2} \cdots, i_{t}\right)$ runs over all sequences $1 \leq i_{1}<i_{2}<\cdots<i_{t} \leq n$ of length $t$ and the product is taken in an arbitrary order. Hence

$$
f \in \operatorname{Im}\left(e: \Gamma^{t} / \Gamma^{t+1} \longrightarrow E_{t}\right)
$$

and the assertion follows.

The James filtration of the tensor algebra $T(V)$

$$
J_{0}(V) \subseteq J_{1}(V) \subseteq \cdots \subseteq J_{n}(V) \subseteq \cdots \subseteq T(V)
$$

induces a cofiltration of the progroup Coalg $(T(-), T(-))$

$$
\cdots \longrightarrow \operatorname{Coalg}\left(J_{n}(-), T(-)\right) \longrightarrow \cdots \longrightarrow \operatorname{Coalg}\left(J_{0}(-), T(-)\right),
$$


where the group Coalg $(T(-), T(-))$ can be identify with the inverse limit

$$
\text { Coalg }(T(-), T(-))=\lim _{n} \operatorname{Coalg}\left(J_{n}(-), T(-)\right) \text {. }
$$

Selick and $\mathrm{Wu}[6]$ proved that there is an isomorphisms of groups

$$
\operatorname{Ker}\left[\operatorname{Coalg}\left(J_{n}(-), T(-)\right) \longrightarrow \operatorname{Coalg}\left(J_{n-1}(-), T(-)\right)\right] \stackrel{\cong}{\longrightarrow} \operatorname{Lie}^{R \otimes \mathbb{Z} / p}(n) .
$$

Definition 4.6 Define the group $\bar{L}_{n}^{R}$ to be the equalizer of the projection maps $\pi_{j}: G\left(A^{R}\left(y_{1}, \ldots y_{n}\right)\right) \longrightarrow G\left(A^{R}\left(y_{1}, \ldots y_{n-1}\right)\right)$.

Lemma 4.7 The group $\bar{L}_{n}^{R}$ is isomorphic to the combinatorial group $\mathcal{H}_{n}^{R}$.

Proof Recall that the isomorphism $K_{n} \cong G\left(A^{R}\left(y_{1}, \ldots y_{n}\right)\right)$ preserves the projection maps, that is, $\exp \circ \pi_{i}=\pi_{i} \circ \exp$. Therefore the assertion of the lemma follows immediately as both groups are given by the equalizer of the same maps.

Theorem 4.8 There is an isomorphism of groups

$$
e: \mathcal{H}_{n}^{R} \longrightarrow \operatorname{Coalg}\left(J_{n}(-), T(-)\right)
$$

for $1 \leq n \leq \infty$.

Proof Let $\theta_{n}: L_{n}^{R} \longrightarrow \operatorname{Hom}_{R}\left(J_{n}(-), T(-)\right)$ be homomorphism (3-1). As the following diagram

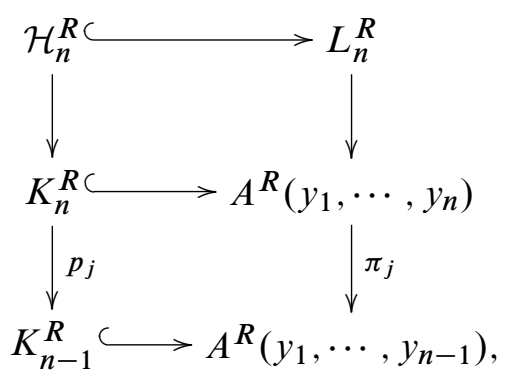

commutes for every $1 \leq j \leq n$ and the bottom two rows are given by inclusions, there is an inclusion $\mathcal{H}_{n}^{R} \longrightarrow L_{n}^{R}$ between the equalizers. Notice that $\theta_{n}\left(\mathcal{H}_{n}^{R}\right) \subseteq$ Coalg $\left(J_{n}(-), T(-)\right)$ and so

$$
e=\left.\theta_{n}\right|_{\mathcal{H}_{n}^{R}}: \mathcal{H}_{n}^{R} \longrightarrow \operatorname{Coalg}\left(J_{n}(-), T(-)\right)
$$

is a well-defined monomorphism of groups. We need to show that $e$ is an epimorphism. 
Recall that there is an isomorphisms of groups

$$
\operatorname{Ker}\left[\operatorname{Coalg}\left(J_{n}(-), T(-)\right) \longrightarrow \operatorname{Coalg}\left(J_{n-1}(-), T(-)\right)\right] \stackrel{\cong}{\longrightarrow} \operatorname{Lie}^{R \otimes \mathbb{Z} / p}(n) .
$$

and that the kernel $\Lambda(n)$ of the homomorphism $d_{n}: \mathcal{H}_{n}^{R} \longrightarrow \mathcal{H}_{n-1}^{R}$ is by Theorem 2.5 isomorphic to $\mathrm{Lie}^{R}(n)$ as a group. Thus there is a commutative diagram

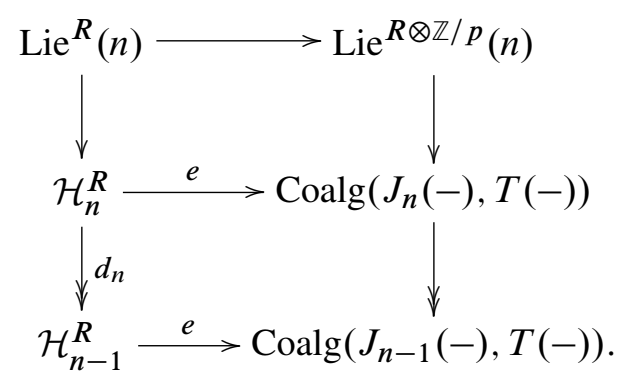

for $1 \leq n \leq \infty$. Therefore, we have that the map $e: \Lambda(n) \cong \operatorname{Lie}^{R}(n) \longrightarrow \operatorname{Lie}^{R \otimes \mathbb{Z} / p}(n)$ is onto. Now by induction, the homomorphism

$$
e: \mathcal{H}_{n}^{R} \longrightarrow \operatorname{Coalg}\left(J_{n}(-), T(-)\right)
$$

is onto for $0 \leq n \leq \infty$. The assertion follows.

Corollary 4.9 There is an isomorphism of groups

$$
e: \mathcal{H}_{\infty}^{R} \longrightarrow \operatorname{Coalg}(T(-), T(-))
$$

Recall that by Theorem 3.10, the combinatorial algebra $L_{n}^{R}$ is isomorphic to $\operatorname{Hom}_{R}\left(J_{n}(-), T(-)\right)$. Therefore we have the following statement.

Corollary 4.10 There is a faithful representation

$$
e: \mathcal{H}_{n}^{R} \longrightarrow L_{n}^{R} \quad \text { for } 1 \leq n \leq \infty .
$$

Theorem 4.11 There is an isomorphism of groups

$$
e:{ }^{R} \mathcal{H}_{n}^{(l)} \longrightarrow \operatorname{Coalg}\left(J_{n}\left(-{ }^{\otimes l}\right), T(-)\right) \text { for } 1 \leq n \leq \infty .
$$

Proof The homomorphism $e:{ }^{R} \mathcal{H}_{n}^{(l)} \longrightarrow \operatorname{Coalg}\left(J_{n}\left(-{ }^{\otimes} \operatorname{Coalg}\left(J_{n}\left(-{ }^{\otimes l}\right), T(-)\right)\right.\right.$ is the homomorphism of the group isomorphisms

$$
e: K_{n}^{R} \longrightarrow \operatorname{Coalg}\left(C(-)^{\otimes n}, T(-)\right),
$$

and thus it is a group isomorphism. 
Recall that by Theorem 3.19 the combinatorial algebra ${ }^{R} L_{n}^{(l)}$ is isomorphic to $\operatorname{Hom}_{R}\left(C(-)^{\otimes n}, T(-)\right)$; hence we have the following corollary.

Corollary 4.12 There is a faithful representation

$$
e:{ }^{R} \mathcal{H}_{n}^{(l)} \longrightarrow{ }^{R} L_{n}^{(l)} \quad \text { for } 1 \leq n \leq \infty .
$$

\section{A representation for the group $K_{n}(k)$}

In this section, we give a representation for the group $K_{n}(k)$.

The algebra $A\left(y_{1}, \cdots, y_{n}\right)$ gives a representation for the group $K_{n}=K_{n}(1)$ (see Section 2). We will use this algebra to give a representation for $K_{n}(k)$ for general $k$, where the representation map into $A\left(y_{1}, \cdots, y_{n}\right)$ depends on $k$.

Recall that the algebra $A\left(y_{1}, \cdots, y_{n}\right)$ is a graded algebra over $\mathbb{Z}$ and $A\left(y_{1}, \cdots, y_{n}\right)_{t}$ is a free $\mathbb{Z}$-module with basis $y_{i_{\sigma(1)}} \cdots y_{i_{\sigma(t)}}$, where $\sigma \in \Sigma_{t}$ acts on $\{1,2, \cdots, t\}$ and $1 \leq i_{1}<i_{2}<\cdots<i_{t} \leq n$.

The (ungraded) commutator in the algebra $A\left(y_{1}, \cdots, y_{n}\right)$ is defined by

$$
[x, y]=x y-y x
$$

for $x, y \in A\left(y_{1}, \cdots, y_{n}\right)$ and the iterated commutator is defined inductively by

$$
\left[\left[x_{1}, \cdots, x_{t}\right]=\left[\left(\left[\left[x_{1}, \cdots, x_{t-1}\right]\right), x_{t}\right] .\right.\right.
$$

Now consider the homogeneous monomial $y_{i_{1}} \cdots y_{i_{k}} \in A\left(y_{1}, \cdots, y_{n}\right)$. Notice that $\left(y_{i_{1}} \cdots y_{i_{k}}\right)^{2}=0$. Thus

$$
\left(1+y_{i_{1}} \cdots y_{i_{k}}\right)\left(1-y_{i_{1}} \cdots y_{i_{k}}\right)=1
$$

and so $1+y_{i_{1}} \cdots y_{i_{k}}$ is a unit element in $A\left(y_{1}, \cdots, y_{n}\right)$. Let $F(k)$ be the free group generated by the words $\left\{x_{i_{1}}|\cdots| x_{i_{k}}\right\}$ with $1 \leq i_{s} \leq n$ for $1 \leq s \leq k$ and let

$$
e: F(k) \longrightarrow A\left(y_{1}, \cdots, y_{n}\right) \text {, given by } e\left(\left\{x_{i_{1}}|\cdots| x_{i_{k}}\right\}\right)=\left(1+y_{i_{1}} \cdots y_{i_{k}}\right),
$$

be a homomorphism from $F(k)$ into the group of units in $A\left(y_{1}, \cdots, y_{n}\right)$. Let $I=$ $\left(i_{1}, \cdots, i_{k}\right)$ be a sequence of integers. Write $x_{I}$ for $\left\{x_{i_{1}}|\cdots| x_{i_{k}}\right\}$ and $y_{I}$ for the monomial $y_{i_{1}} \cdots y_{i_{k}}$.

Theorem 5.1 The representation map e: $F(k) \longrightarrow A\left(y_{1}, \cdots, y_{n}\right)$ factors through the quotient group $K_{n}(k)$. Furthermore, the resulting representation map

$$
e: K_{n}(k) \longrightarrow A\left(y_{1}, \cdots, y_{n}\right)
$$

is faithful. 
The proof requires the following lemma.

\section{Lemma 5.2}

(1) Let $w=x_{I_{1}}^{n_{1}} \cdots x_{I_{t}}^{n_{t}}$ be a word in $F(k)$. Then

$$
e(w)=\left(1+n_{1} y_{I_{1}}\right) \cdots\left(1+n_{t} y_{I_{t}}\right) .
$$

(2) $e\left(\left[\left[x_{I_{1}}^{n_{1}}, \cdots, x_{I_{t}}^{n_{t}}\right]\right)=1+n_{1} \cdots n_{t}\left[\left[y_{I_{1}}, \cdots, y_{I_{t}}\right]\right.\right.$, where the first bracket $[[\cdots]$ is the commutator in the group $F(k)$ and the second bracket $[[\cdots]$ is the commutator in the algebra $A\left(y_{1}, \cdots, y_{n}\right)$.

Proof Notice that $\left(1+n y_{I}\right)\left(1-n y_{I}\right)=1-n^{2}\left(y_{I}\right)^{2}=1$ for $n \in \mathbb{Z}$. Thus

$$
e\left(x_{I}^{-n}\right)=1-n y_{I}
$$

and so assertion (1) follows.

The proof of assertion (2) is given by induction on the length $t$. Let

$$
\begin{aligned}
w & =\left[\left[x_{I_{1}}^{n_{1}}, \cdots, x_{I_{t}}^{n_{t}}\right]\right. \\
\text { with } t \geq 2 \text { and let } \quad w^{\prime} & =\left[\left[x_{I_{1}}^{n_{1}}, \cdots, x_{I_{t-1}}^{n_{t-1}}\right]\right.
\end{aligned}
$$

Notice that $w=\left(w^{\prime}\right)^{-1} x_{I_{t}}^{-n_{t}} w^{\prime} x_{I_{t}}^{n_{t}}$. By induction, one has

$$
e\left(w^{\prime}\right)=1+n_{1} \cdots n_{t-1}\left[\left[y_{I_{1}}, \cdots, y_{I_{t-1}}\right]\right.
$$

and so $\quad e\left(\left(w^{\prime}\right)^{-1}\right)=1-n_{1} \cdots n_{t-1}\left[\left[y_{I_{1}}, \cdots, y_{I_{t-1}}\right]\right.$

because $\left[\left[y_{I_{1}}, \cdots, y_{I_{t-1}}\right]^{2}=0\right.$ in the algebra $A\left(y_{1}, \cdots, y_{n}\right)$. Thus

$$
\begin{aligned}
& e(w)= \\
& \left(1-n_{1} \cdots n_{t-1}\left[\left[y_{I_{1}}, \cdots, y_{I_{t-1}}\right]\right)\left(1-n_{t} y_{I_{t}}\right)\left(1+n_{1} \cdots n_{t-1}\left[\left[y_{I_{1}}, \cdots, y_{I_{t-1}}\right]\right)\left(1+n_{t} y_{I_{t}}\right) .\right.\right.
\end{aligned}
$$

It is routine to check that the right side in the above equation is equal to

$$
1+n_{1} \cdots n_{t}\left[\left[y_{I_{1}}, \cdots, y_{I_{t}}\right]\right.
$$

in the algebra $A\left(y_{1}, \cdots, y_{n}\right)$. Assertion (2) follows.

Proof of Theorem 5.1 Let $I=\left(i_{1}, \cdots, i_{k}\right)$. Then $e\left(x_{I}\right)=1+y_{i_{1}} \cdots y_{i_{k}}$. If $i_{p}=i_{q}$ for some $p<q$, then $y_{i_{1}} \cdots y_{i_{k}}=0$ and so the representation map $e$ preserves relation (1) in the definition of $K_{n}(k)$.

Let $I_{j}=\left(i_{j 1}, \cdots, i_{j k}\right)$ with $1 \leq j \leq t$. Then

$$
e\left(\left[\left[x_{I_{1}}, \cdots, x_{I_{t}}\right]\right)=1+\left[\left[y_{I_{1}}, \cdots, y_{I_{t}}\right] .\right.\right.
$$


Notice that

$$
\left[\left[y_{I_{1}}, \cdots, y_{I_{t}}\right]=\sum_{\sigma} \pm y_{I_{\sigma(1)}} \cdots y_{I_{\sigma(t)}},\right.
$$

where $\sigma$ runs over certain elements in $\Sigma_{t}$.

Suppose that $i_{p q}=i_{p^{\prime} q^{\prime}}$ for some $(p, q) \neq\left(p^{\prime}, q^{\prime}\right)$. Then

$$
\left[\left[y_{I_{1}}, \cdots, y_{I_{t}}\right]=0\right.
$$

and so $e\left(\left[\left[x_{I_{1}}, \cdots, x_{I_{t}}\right]\right)=1\right.$. That is, the representation map $e$ preserves relation (2) in the definition of $K_{n}(k)$.

Now it is easy to check that

$$
\text { Thus } \begin{aligned}
\left(1+\left[\left[y_{I_{1}}, \cdots, y_{I_{t}}\right]\right)^{n}\right. & =1+n\left[\left[y_{I_{1}}, \cdots, y_{I_{t}}\right] .\right. \\
e\left(\left[\left[x_{I_{1}}^{n_{1}}, \cdots, x_{I_{t}}^{n_{t}}\right]\right)\right. & =1+n_{1} \cdots n_{t}\left[\left[y_{I_{1}}, \cdots, y_{I_{t}}\right]\right. \\
& =\left(1+\left[\left[y_{I_{1}}, \cdots, y_{I_{t}}\right]\right)^{n_{1} \cdots n_{t}}=e\left(\left[\left[x_{I_{1}}, \cdots, x_{I_{t}}\right]^{n_{1} \cdots n_{t}}\right)\right.\right.
\end{aligned}
$$

and so the representation map $e$ preserves relation (3) in the definition of $K_{n}(k)$. Thus the representation map $e: F(k) \longrightarrow A\left(y_{1}, \cdots, y_{n}\right)$ factors through $K_{n}(k)$, which is the first statement in the theorem.

Now we need to show that the representation map $e: K_{n}(k) \longrightarrow A\left(y_{1}, \cdots, y_{n}\right)$ is faithful. Let $A(k)$ be the graded subalgebra of $A\left(y_{1}, \cdots, y_{n}\right)$ generated by all the elements $\alpha \in A\left(y_{1}, \cdots, y_{n}\right)_{k}$ of dimension $k$. Notice that the image of the representation map $e$ is contained in the subalgebra $A(k)$. That is,

$$
e: K_{n}(k) \longrightarrow A(k)
$$

is a representation map. Let $I(A(k))$ be the augmentation ideal of the algebra $A(k)$ and let $\theta: K_{n}(k) \longrightarrow I(A(k))$ be the function $\theta(w)=e(w)-1$ for $w \in K_{n}(k)$. Let $\left\{\Gamma^{r}(G)\right\}$ be the lower central series of a group $G$ and let $I^{t}(A)=(I(A))^{t}$ be the $t$-fold product of the augmentation ideal $I(A)$ of an augmented algebra $A$. To finish the proof the following two lemmas are required.

Lemma 5.3 Let $G$ be a group and let $e: G \longrightarrow A$ be a representation of $G$ into an augmented algebra $A$ over $\mathbb{Z}$ such that $\epsilon(e(g))=1$ for $g \in G$, where $\epsilon: A \longrightarrow \mathbb{Z}$ is the augmentation map. Let $\theta: G \rightarrow I(A)$ be the function $\theta(g)=g-1$ for all $g \in G$. Then

(1) $\theta\left(\Gamma^{t}(G)\right) \subseteq I^{t}(A)$ for each $t \geq 1$.

(2) $\theta$ induces a homomorphism of abelian groups

$$
\bar{\theta}: \Gamma^{t}(G) / \Gamma^{t+1}(G) \longrightarrow I^{t}(A) / I^{t+1}(A)
$$


such that the diagram

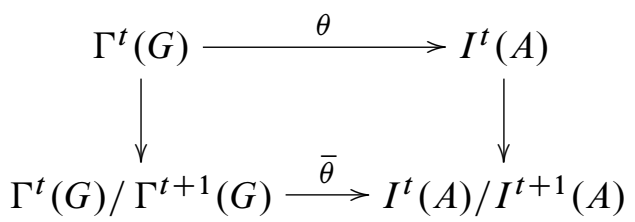

commutes.

(3) The map

$$
\bar{\theta}: \sum_{t \geq 1} \Gamma^{t}(G) / \Gamma^{t+1}(G) \longrightarrow \sum_{t \geq 0} I^{t}(A) / I^{t+1}(A)
$$

is a representation map of the Lie algebra $\sum_{t \geq 1} \Gamma^{t}(G) / \Gamma^{t+1}(G)$ into the algebra $\sum_{t \geq 0} I^{t}(A) / I^{t+1}(A)$.

Lemma 5.4 The map

$$
\bar{\theta}: \Gamma^{t}\left(K_{n}(k)\right) / \Gamma^{t+1}\left(K_{n}(k)\right) \longrightarrow I^{t}(A(k)) / I^{t+1}(A(k))
$$

is a monomorphism for each $t \geq 1$.

Continuation of the Proof of Theorem 5.1 (given Lemma 5.3 and Lemma 5.4)

By relation (2) in the definition of the group $K_{n}(k)$, the group $K_{n}(k)$ is nilpotent and so $\Gamma^{s}\left(K_{n}(k)\right)$ is trivial for some $s$. Suppose that $e: \Gamma^{t+1}\left(K_{n}(k)\right) \longrightarrow A(k)$ is faithful. That is, $w=1$ if $e(w)=1$ and $w \in \Gamma^{t+1}\left(K_{n}(k)\right)$. Let $w \in \Gamma^{t}\left(K_{n}(k)\right)$ be such that $e(w)=1$. Then $\theta(w)=0$ and so $\bar{\theta}(\bar{w})=0$ where $\bar{w}$ is the image of $w$ in $\Gamma^{t}\left(K_{n}(k)\right) / \Gamma^{t+1}\left(K_{n}(k)\right)$. By Lemma 5.4, $\bar{w}$ is zero in the abelian group $\Gamma^{t}\left(K_{n}(k)\right) / \Gamma^{t+1}\left(K_{n}(k)\right)$ and so $w \in \Gamma^{t+1}\left(K_{n}(k)\right)$. By induction, $w=1$ and the assertion follows.

Proof of Lemma 5.3 Let $\tilde{e}: \mathbb{Z}(G) \longrightarrow A$ be the map of algebras such that $\tilde{e}(g)=$ $e(g)$, where $\mathbb{Z}(G)$ is the group ring of $G$ over $\mathbb{Z}$. Notice that $\epsilon(e(g))=1$ for $g \in G$. The map $\tilde{e}: \mathbb{Z}(G) \longrightarrow A$ is a morphism of augmented algebras. Let $\theta^{\prime}: G \longrightarrow I(\mathbb{Z}(G))$ be the function $\theta^{\prime}(g)=g-1$ for $g \in G$. Then $\theta=\tilde{e} \circ \theta^{\prime}: G \longrightarrow A$. Notice that the assertions hold for the function $\theta^{\prime}: G \longrightarrow \mathbb{Z}(G)$, (see, for example, [3, Proposition 10.5, page 187]), and $\tilde{e}$ is a morphism of augmented algebras. The assertion follows.

We need two more lemmas to prove Lemma 5.4. 
Lemma 5.5 [5] Let $A$ be an (ungraded) algebra over $\mathbb{Z}$ and let $x_{1}, \cdots, x_{n}$ be elements in $A$. Then

$$
\left[\left[x_{1}, \cdots, x_{n}\right]=\sum_{0 \leq p \leq n-1}(-1)^{p} x_{i_{p}} \cdots x_{i_{1}} x_{j_{1}} \cdots x_{j_{n-p}},\right.
$$

where the sum is taken over $0 \leq p \leq n-1$ and all shuffles $\left(i_{1}, \cdots, i_{p} ; j_{1}, \cdots, j_{n-p}\right)$ such that:

(1) $1 \leq i_{1}<i_{2}<\cdots<i_{p} \leq n$;

(2) $1 \leq j_{1}<j_{2}<\cdots<j_{n-p} \leq n$;

(3) $\left\{i_{1}, \cdots, i_{p}\right\} \cap\left\{j_{1}, \cdots, j_{n-p}\right\}=\varnothing$.

Proof The proof is given by induction on the length $n$. The assertion holds trivially for the case $n=2$. Suppose that the assertion holds for $n-1$. Then

$$
\begin{gathered}
{\left[\left[x_{1}, \cdots, x_{n}\right]=\left[\left[x_{1}, \cdots, x_{n-1}\right] x_{n}-x_{n}\left[\left[x_{1}, \cdots, x_{n-1}\right]\right.\right.\right.} \\
=\sum_{0 \leq p \leq n-2}(-1)^{p} x_{i_{p}} \cdots x_{i_{1}} x_{j_{1}} \cdots x_{j_{n-p-1}} x_{n}-\sum_{0 \leq p \leq n-2}(-1)^{p} x_{n} x_{i_{p}} \cdots x_{i_{1}} x_{j_{1}} \cdots x_{j_{n-p-1}} \\
=\sum_{0 \leq p \leq n-2 ; i_{p}<n}(-1)^{p} x_{i_{p}} \cdots x_{i_{1}} x_{j_{1}} \cdots x_{j_{n-p-1}} x_{n}+\sum_{1 \leq p \leq n-1 ; i_{p}=n}(-1)^{p} x_{i_{p}} \cdots x_{i_{1}} x_{j_{1}} \cdots x_{j_{n-p}} .
\end{gathered}
$$

Notice that $x_{i_{p}} \cdots x_{i_{1}} x_{j_{1}} \cdots x_{j_{n-p}}=x_{1} \cdots x_{n}$ or $x_{n} \cdots x_{1}$ if $p=0$ or $n-1$, respectively. The assertion follows.

A sequence of integers $I=\left(i_{1}, \cdots, i_{s}\right)$ is called $n$-admissible if $i_{p} \neq i_{q}$ for all $p<q$ and $1 \leq i_{j} \leq n$ for $1 \leq j \leq s$. The length $s$ of the sequence $I=\left(i_{1}, \cdots, i_{s}\right)$ will be denoted by $l(I)$. Let $I_{j}=\left(i_{j 1}, \cdots, i_{j k_{j}}\right)$ be sequences of integers with $1 \leq j \leq t$. The sequence (of sequences) $\left(I_{1}, \cdots, I_{t}\right)$ is called $n$-admissible if the sequence $\left(i_{11}, \cdots, i_{1 k_{1}}, \cdots, i_{t 1}, \cdots, i_{t k_{t}}\right)$ is $n$-admissible. Now consider the graded algebra $A(k)$. Let $T\left(y_{I}\right)$ be the tensor algebra over $\mathbb{Z}$ generated by the words $y_{I}$, where $I=\left(i_{1}, \cdots, i_{k}\right)$ is a sequence such that $1 \leq i_{j} \leq n$ for $1 \leq j \leq k$. Let $q: T\left(y_{I}\right) \longrightarrow$ $A(k)$ be the quotient map of algebras, where $q\left(y_{I}\right)=y_{I}$. Let $L\left(y_{I}\right) \subseteq T\left(y_{I}\right)$ be the associated Lie algebra of $T\left(y_{I}\right)$. Let $A_{t}(k)$ denote the image $q\left(T_{t}\left(y_{I}\right)\right)$, let $\bar{L}(A(k))$ denote $q\left(L\left(y_{I}\right)\right)$ and let $\bar{L}_{t}(A(k))$ denote $q\left(L_{t}\left(y_{I}\right)\right)$ where $L_{t}\left(y_{I}\right)$ is spanned by the Lie elements of tensor length $t$ in the Lie algebra $L\left(y_{I}\right)$. Let $\mathcal{S}_{k}$ be the set of the $n$-admissible sequences of length $k$ with some chosen order $<$. 


\section{Lemma 5.6}

(1) A basis for the free $\mathbb{Z}$-module $A_{t}(k)=A\left(y_{1}, \cdots, y_{n}\right)_{k t}$ is given by the monomials $y_{I_{1}} \cdots y_{I_{t}}$, where $I_{j} \in \mathcal{S}_{k}$ for $1 \leq j \leq t$ and the sequence $\left(I_{1}, \cdots, I_{t}\right)$ is admissible.

(2) A basis for the free $\mathbb{Z}$-module $\bar{L}_{t}(A(k))$ is given by the Lie elements

$$
\left[\left[y_{I_{1}}, y_{I_{\sigma(2)}}, \cdots, y_{I_{\sigma(t)}}\right]\right.
$$

where $I_{j} \in \mathcal{S}_{k}$ for $1 \leq j \leq t$ such that $I_{1}<I_{2}<\cdots<I_{t}$ and where $\left(I_{1}, \cdots, I_{t}\right)$ is admissible and $\sigma \in \Sigma_{t-1}$ acts on $\{2, \cdots, t\}$.

Proof It is easy to check that the set of the monomials

$$
\left\{y_{I_{1}} \cdots y_{I_{t}} \mid I_{j} \in \mathcal{S}_{k} \text { for } 1 \leq j \leq t \text { and }\left(I_{1}, \cdots, I_{t}\right) \text { is admissible }\right\}
$$

is the same as that of monomials

$$
\left\{y_{i_{1}} \cdots y_{i_{k t}} \mid\left(i_{1}, \cdots, i_{k t}\right) \text { is admissible }\right\} .
$$

Now the latter is a basis for $A\left(y_{1}, \cdots, y_{n}\right)_{k t}=A_{t}(k)$. Assertion (1) follows.

For assertion (2), it suffices to show that the Lie elements given in assertion (2) are linearly independent because every element in $\bar{L}_{t}(A(k))$ is a linear combination of such elements.

Let $I_{j}, J_{j} \in \mathcal{S}_{k}$ for $1 \leq j \leq t$ such that:

(1) $I_{1}<\cdots<I_{t}, J_{1}<\cdots<J_{t}$

(2) both $\left(I_{1}, \cdots, I_{t}\right)$ and $\left(J_{1}, \cdots, J_{t}\right)$ are admissible.

Let $\sigma, \tau \in \Sigma_{t-1}$. By assertion $(1), \operatorname{Hom}_{\mathbb{Z}}\left(A_{t}(k), \mathbb{Z}\right)$ has a dual basis. Consider

$$
\left\langle\left[\left[y_{J_{1}}, y_{J_{\tau(2)}}, \cdots, y_{J_{\tau(t)}}\right],\left(y_{I_{1}} y_{I_{\sigma(2)}} \cdots y_{I_{\sigma(t)}}\right)^{*}\right\rangle .\right.
$$

If the set $\left\{J_{1}, \cdots, J_{t}\right\} \neq\left\{I_{1}, \cdots, I_{t}\right\}$, then it is easy to check that

$$
\left\langle\left[\left[y_{J_{1}}, y_{J_{\tau(2)}}, \cdots, y_{J_{\tau(t)}}\right],\left(y_{I_{1}} y_{I_{\sigma(2)}} \cdots y_{I_{\sigma(t)}}\right)^{*}\right\rangle=0 .\right.
$$

If $\left\{J_{1}, \cdots, J_{t}\right\}=\left\{I_{1}, \cdots, I_{t}\right\}$ as a set, then $J_{j}=I_{j}$ for all $1 \leq j \leq t$. By Lemma 5.5 ,

$$
\left[\left[y_{J_{1}}, y_{J_{\tau(2)}}, \cdots, y_{J_{\tau(t)}}\right]=y_{J_{1}} y_{J_{\tau(2)}} \cdots y_{J_{\tau(t)}}+\sum \pm y_{K_{1}} y_{K_{2}} \cdots y_{K_{t}},\right.
$$


where $\sum \pm y_{K_{1}} y_{K_{2}} \cdots y_{K_{t}}$ is the sum of the other terms in Lemma 5.5. Notice that $y_{K_{1}} \neq y_{J_{1}}$ in each term of the sum $\sum y_{K_{1}} y_{K_{2}} \cdots y_{K_{t}}$. Thus the scalar product

$$
\left\langle\left[\left[y_{J_{1}}, y_{J_{\tau(2)}}, \cdots, y_{J_{\tau(t)}}\right],\left(y_{I_{1}} y_{I_{\sigma(2)}} \cdots y_{I_{\sigma(t)}}\right)^{*}\right\rangle=\left\{\begin{array}{l}
1 \text { for }\left(J_{1}, \cdots, J_{t}\right)=\left(I_{1}, \cdots, I_{t}\right), \\
\text { and } \tau=\sigma \\
0 \text { otherwise. }
\end{array}\right.\right.
$$

Assertion (2) now follows easily.

Proof of Lemma 5.4 By Lemma 5.2, the $\mathbb{Z}$-map

$$
\bar{\theta}: \Gamma^{t}\left(K_{n}(k)\right) / \Gamma^{t+1}\left(K_{n}(k)\right) \longrightarrow I^{t}(A(k)) / I^{t+1}(A(k))=A_{t}(k)
$$

maps onto the $\mathbb{Z}$-submodule $\bar{L}_{t}(A(k))$. By relation (2) in the definition of the group $K_{n}(k)$, every element in the abelian group $\Gamma^{t}\left(K_{n}(k)\right) / \Gamma^{t+1}\left(K_{n}(k)\right)$ is a linear combination of the elements $\overline{\left[\left[x_{I_{1}}, x_{I_{\sigma(2)}}, \cdots, x_{I_{\sigma(t)}}\right]\right.}$, where $I_{j} \in \mathcal{S}_{k}$ for $1 \leq j \leq t$, $\left(I_{1}<\cdots<I_{t}\right)$ is admissible and $\sigma \in \Sigma_{t-1}$ acts on $\{2, \cdots, t\}$. Thus the $\mathbb{Z}$-map $\phi: \bar{L}_{t}(A(k)) \longrightarrow \Gamma^{t}\left(K_{n}(k)\right) / \Gamma^{t+1}\left(K_{n}(k)\right)$ defined by

$$
\phi\left(\left[\left[y_{I_{1}}, y_{I_{\sigma(2)}}, \cdots, y_{I_{\sigma(t)}}\right]\right)=\overline{\left[\left[x_{I_{1}}, x_{I_{\sigma(2)}} \cdots, x_{I_{\sigma(t)}}\right]\right.}\right.
$$

is an epimorphism. Notice that the composite

$$
\bar{L}_{t}(A(k)) \stackrel{\phi}{\longrightarrow} \Gamma^{t}\left(K_{n}(k)\right) / \Gamma^{t+1}\left(K_{n}(k)\right) \stackrel{\bar{\theta}}{\longrightarrow} \bar{L}_{t}(A(k))
$$

is an isomorphism. Thus

$$
\phi: \bar{L}_{t}(A(k)) \longrightarrow \Gamma^{t}\left(K_{n}(k)\right) / \Gamma^{t+1}\left(K_{n}(k)\right)
$$

is a monomorphism and hence it is an isomorphism. The same argument proves that

$$
\bar{\theta}: \Gamma^{t}\left(K_{n}(k)\right) / \Gamma^{t+1}\left(K_{n}(k)\right) \longrightarrow \bar{L}_{t}(A(k))
$$

is an isomorphism. The assertion follows.

Consider the composite

$$
\bar{e}: K_{n}(k) \stackrel{e}{\longrightarrow} A\left(y_{1}, \cdots, y_{n}\right) \stackrel{q}{\longrightarrow} A^{\mathbb{Z} / p^{r}}\left(y_{1}, \cdots, y_{n}\right) .
$$

Notice that, in the algebra $A^{\mathbb{Z} / p^{r}}\left(y_{1}, \cdots, y_{n}\right)$, we have

$$
\left(1+y_{i_{1}} \cdots y_{i_{k}}\right)^{p^{r}}=1+p^{r} y_{i_{1}} \cdots y_{i_{k}}=1 .
$$

Thus the representation map $\bar{e}: K_{n}(k) \longrightarrow A^{\mathbb{Z} / p^{r}}\left(y_{1}, \cdots, y_{n}\right)$ factors through the quotient group $K_{n}^{\mathbb{Z} / p^{r}}(k)$. 
Proposition 5.7 The representation map $\bar{e}: K_{n}^{\mathbb{Z} / p^{r}}(k) \longrightarrow A^{\mathbb{Z} / p^{r}}\left(y_{1}, \cdots, y_{n}\right)$ is faithful.

Proof Let $A^{\mathbb{Z} / p^{r}}(k)=A(k) \otimes Z / p^{r}$. It suffices to show that

$\bar{\theta}: \Gamma^{t}\left(K_{n}^{\mathbb{Z} / p^{r}}(k)\right) / \Gamma^{t+1}\left(K_{n}^{\mathbb{Z} / p^{r}}(k)\right)$

$$
\longrightarrow I^{t}\left(A^{\mathbb{Z} / p^{r}}(k)\right) / I^{t+1}\left(A^{\mathbb{Z} / p^{r}}(k)\right)=A_{t}(k) \otimes \mathbb{Z} / p^{r}
$$

is a monomorphism. Notice that

$$
\bar{\theta}: \Gamma^{t}\left(K_{n}^{\mathbb{Z} / p^{r}}(k)\right) / \Gamma^{t+1}\left(K_{n}^{\mathbb{Z} / p^{r}}(k)\right) \longrightarrow I^{t}\left(A^{\mathbb{Z} / p^{r}}(k)\right) / I^{t+1}\left(A^{\mathbb{Z} / p^{r}}(k)\right)
$$

maps onto the submodule $\bar{L}_{t}(A(k)) \otimes \mathbb{Z} / p^{r}$. Observe that $L_{t}(A(k)) \otimes \mathbb{Z} / p^{r}$ is a free $\mathbb{Z} / p^{r}$-module with a basis $\left[\left[y_{I_{1}}, y_{I_{\sigma(2)}}, \cdots, y_{I_{\sigma(t)}}\right]\right.$, where $I_{j} \in \mathcal{S}_{k}$ for $1 \leq j \leq t$, where $\left(I_{1}<\cdots<I_{t}\right)$ is admissible and where $\sigma \in \Sigma_{t-1}$ acts on $\{2, \cdots, t\}$. By the definition of $K_{n}^{\mathbb{Z} / p^{r}}(k)$, the map

$$
\phi: L_{t}(A(k)) \otimes \mathbb{Z} / p^{r} \longrightarrow \Gamma^{t}\left(K_{n}^{\mathbb{Z} / p^{r}}(k)\right) / \Gamma^{t+1}\left(K_{n}^{\mathbb{Z} / p^{r}}(k)\right)
$$

given by

$$
\phi\left(\left[\left[y_{I_{1}}, y_{I_{\sigma(2)}} \cdots, y_{I_{\sigma(t)}}\right]\right)=\overline{\left[\left[x_{I_{1}}, x_{I_{\sigma(2)}} \cdots, x_{I_{\sigma(t)}}\right]\right.}\right.
$$

is a well-defined epimorphism. The assertion follows from the fact that the composite $\bar{\theta} \circ \phi$ is an isomorphism.

Theorem 5.8 There is an isomorphism of groups

$$
e: K_{n}^{R}(k) \longrightarrow \operatorname{Coalg}\left(C(-)^{\otimes n}, T\left(-^{\wedge k}\right)\right) \quad \text { for } 1 \leq n \leq \infty .
$$

Proof The proof follows along the lines of the proof of Theorem 4.8.

Theorem 5.9 There is an isomorphism of groups

$$
e:{ }^{R} \mathcal{H}_{n}^{(l),(k)} \longrightarrow \operatorname{Coalg}\left(J_{n}\left(-^{\wedge l}\right), T\left(-^{\wedge k}\right)\right) \quad \text { for } 1 \leq n \leq \infty .
$$

Proof The homomorphism $e:{ }^{R} \mathcal{H}_{n}^{(l)(k)} \rightarrow \operatorname{Coalg}\left(J_{n}\left(-^{\wedge} \operatorname{Coalg}\left(J_{n}\left(-^{\wedge} l\right), T\left(-^{\wedge k}\right)\right)\right.\right.$ is the by the group isomorphisms

$$
e: K_{n}^{R}(k) \longrightarrow \operatorname{Coalg}\left(C(-)^{\otimes n}, T\left(-^{\wedge k}\right)\right),
$$

and thus it is a group isomorphism.

Corollary 5.10 There is a faithful representation

$$
e:{ }^{R} \mathcal{H}_{n}^{(l),(k)} \longrightarrow{ }^{R} L_{n}^{(l),(k)} \text { for } 1 \leq n \leq \infty .
$$




\section{References}

[1] F R Cohen, On combinatorial group theory in homotopy theory, preprint

[2] F R Cohen, On combinatorial group theory in homotopy, from: "Homotopy theory and its applications (Cocoyoc, 1993)", Contemp. Math. 188, Amer. Math. Soc., Providence, RI (1995) 57-63 MR1349129

[3] E B Curtis, Simplicial homotopy theory, Advances in Math. 6 (1971) 107-209 (1971) MR0279808

[4] J Grbić, J Wu, Applications of combinatorial groups to Hopf invariant and the exponent problem, Algebr. Geom. Topol. 6 (2006) 2229-2255

[5] C Reutenauer, Free Lie algebras, London Mathematical Society Monographs. New Series 7, Oxford Science Publications, Oxford University Press (1993) MR1231799

[6] P Selick, J Wu, On natural coalgebra decompositions of tensor algebras and loop suspensions, Mem. Amer. Math. Soc. 148 (2000) MR1706247

Department of Mathematical Sciences, University of Aberdeen

Meston Building, Aberdeen AB24 3UE, UK

Department of Mathematics, National University of Singapore

2 Science Drive 2, Singapore

jelena@maths.abdn.ac.uk, matwuj@nus.edu.sg

Received: 23 October 2006 